\title{
On counting special Lagrangian homology 3-spheres
}

\author{
Dominic Joyce \\ Lincoln College, Oxford, OX1 3DR
}

\section{Introduction}

It is well known [14] that one can define Gromov-Witten invariants of symplectic manifolds $(M, \omega)$ by choosing a generic compatible metric $g$ and almost complex structure $J$ on $M$, and counting the $J$-holomorphic curves $\Sigma$ in $M$ in a given homology class, with signs. The invariants are then essentially independent of the choice of $g, J$. As $J$-holomorphic curves are calibrated 2submanifolds with respect to the calibration $\omega$ on the Riemannian manifold $(M, g)$, Gromov-Witten invariants arise by counting the simplest nontrivial kind of calibrated submanifold.

In this paper we shall attempt to define a similar invariant $I$ of generic (almost) Calabi-Yau 3-folds $(M, J, \omega, \Omega)$ by counting another kind of calibrated submanifold, special Lagrangian 3-submanifolds, or SL 3-folds for short. In fact we shall consider only special Lagrangian rational homology 3-spheres, as they occur in 0-dimensional moduli spaces, and can be counted. Let $S(\delta)$ be the set of SL homology 3-spheres in $M$ with homology class $\delta$. Suppose $S(\delta)$ is finite. Then we shall define

$$
I(\delta)=\sum_{N \in S(\delta)} w(N)
$$

where $w(N)$ is a rational 'weight function' depending on the topology of $N$.

For $I$ to be interesting it should either be unchanged by smooth deformations of the underlying almost Calabi-Yau structure $(J, \omega, \Omega)$, or else should transform according to some rigid set of rules as $[\omega]$ and $[\Omega]$ move about in $H^{2}(M, \mathbb{R})$ and $H^{3}(M, \mathbb{C})$. Now whether such an invariant can be made 
to work, and how it should be defined, depends very much on the singular behaviour of special Lagrangian 3-folds, which is not well understood. Therefore much of this paper is conjectural, though I hope to be able to publish proofs of many of the conjectures in the next few years, unless someone else does first.

Here is why singularities of SL 3-folds are important in this problem. In the moduli space of almost Calabi-Yau structures on $M$ there are certain special real hypersurfaces, determined using the homology of $M$. At such a hypersurface, some of the SL 3-folds in $M$ will become singular. An SL 3-fold may exist in $M$ only on one side of the hypersurface, and become singular at the hypersurface.

More generally, as we approach the hypersurface from one side, one or more SL 3-folds may collapse down to a singular SL 3-fold, and then on the other side this singular SL 3-fold is replaced by a different collection of one or more SL 3-folds. The key question addressed in this paper is to find a way to define the invariant $I$ so that it is unchanged, or transforms in a controlled way, as we cross these hypersurfaces and the set of SL 3-folds that we are 'counting' changes.

In $\S 3-\delta$ and $\S-\delta 6$ we study two kinds of singularity that SL 3-folds can develop, modelled respectively on a $T^{2}$-cone in $\mathbb{C}^{3}$, and the union of two SL 3-planes $\mathbb{R}^{3}$ in $\mathbb{C}^{3}$. We use these transitions to calculate identities which the weight function $w(N)$ in (1) must satisfy for $I$ to be invariant, or transform nicely, as we pass through the hypersurface. It turns out that the simple weight function $w(N)=\left|H_{1}(N, \mathbb{Z})\right|$ satisfies these identities.

Motivated by this, we formulate a conjecture, Conjecture 7.3, giving a partial definition of $I$, and a partial statement of the transformation law it should satisfy under deformation. A full definition and transformation law (if the invariant works at all) will have to await a better understanding of singular SL 3-folds. We conclude in 87.6 with a discussion of the relationship of the invariants to String Theory. The author believes that they count objects of significance in String Theory, namely isolated 3-branes, and that they should play a rôle in Mirror Symmetry of Calabi-Yau 3-folds.

Acknowledgements. I would like to thank Bobby Acharya, Edward Witten, Simon Donaldson, and Mark Gross for helpful conversations. 


\section{Special Lagrangian geometry}

We now introduce the idea of special Lagrangian submanifolds (SL $m$-folds), in two different geometric contexts. First, in $\$ 2.1$, we define SL $m$-folds in $\mathbb{C}^{m}$. Then $\$ 2.2$ discusses SL $m$-folds in almost Calabi-Yau m-folds, compact Kähler manifolds equipped with a holomorphic volume form which generalize the idea of Calabi-Yau manifolds. Finally, section 2.3 considers the singularities of SL $m$-folds. The principal references for this section are Harvey and Lawson [5] and the author [12].

\subsection{Special Lagrangian submanifolds in $\mathbb{C}^{m}$}

We begin by defining calibrations and calibrated submanifolds, following Harvey and Lawson [5].

Definition 2.1 Let $(M, g)$ be a Riemannian manifold. An oriented tangent $k$-plane $V$ on $M$ is a vector subspace $V$ of some tangent space $T_{x} M$ to $M$ with $\operatorname{dim} V=k$, equipped with an orientation. If $V$ is an oriented tangent $k$-plane on $M$ then $\left.g\right|_{V}$ is a Euclidean metric on $V$, so combining $\left.g\right|_{V}$ with the orientation on $V$ gives a natural volume form $\operatorname{vol}_{V}$ on $V$, which is a $k$-form on $V$.

Now let $\varphi$ be a closed $k$-form on $M$. We say that $\varphi$ is a calibration on $M$ if for every oriented $k$-plane $V$ on $M$ we have $\left.\varphi\right|_{V} \leq \operatorname{vol}_{V}$. Here $\left.\varphi\right|_{V}=\alpha \cdot \operatorname{vol}_{V}$ for some $\alpha \in \mathbb{R}$, and $\left.\varphi\right|_{V} \leq \operatorname{vol}_{V}$ if $\alpha \leq 1$. Let $N$ be an oriented submanifold of $M$ with dimension $k$. Then each tangent space $T_{x} N$ for $x \in N$ is an oriented tangent $k$-plane. We say that $N$ is a calibrated submanifold if $\left.\varphi\right|_{T_{x} N}=\operatorname{vol}_{T_{x} N}$ for all $x \in N$.

It is easy to show that calibrated submanifolds are automatically minimal submanifolds [5, Th. II.4.2]. Here is the definition of special Lagrangian submanifolds in $\mathbb{C}^{m}$, taken from [5, §III].

Definition 2.2 Let $\mathbb{C}^{m}$ have complex coordinates $\left(z_{1}, \ldots, z_{m}\right)$, and define a metric $g$, a real 2 -form $\omega$ and a complex $m$-form $\Omega$ on $\mathbb{C}^{m}$ by

$$
\begin{aligned}
g=\left|\mathrm{d} z_{1}\right|^{2}+\cdots+\left|\mathrm{d} z_{m}\right|^{2}, & \omega=\frac{i}{2}\left(\mathrm{~d} z_{1} \wedge \mathrm{d} \bar{z}_{1}+\cdots+\mathrm{d} z_{m} \wedge \mathrm{d} \bar{z}_{m}\right), \\
\text { and } \quad & \Omega=\mathrm{d} z_{1} \wedge \cdots \wedge \mathrm{d} z_{m} .
\end{aligned}
$$

Then $\operatorname{Re} \Omega$ and $\operatorname{Im} \Omega$ are real $m$-forms on $\mathbb{C}^{m}$. Let $L$ be an oriented real submanifold of $\mathbb{C}^{m}$ of real dimension $m$. We say that $L$ is a special Lagrangian 
submanifold of $\mathbb{C}^{m}$, or $S L m$-fold for short, if $L$ is calibrated with respect to $\operatorname{Re} \Omega$, in the sense of Definition 2.1.

Harvey and Lawson [5, Cor. III.1.11] give the following alternative characterization of special Lagrangian submanifolds:

Proposition 2.3 Let $L$ be a real $m$-dimensional submanifold of $\mathbb{C}^{m}$. Then $L$ admits an orientation making it into an SL submanifold of $\mathbb{C}^{m}$ if and only if $\left.\omega\right|_{L} \equiv 0$ and $\left.\operatorname{Im} \Omega\right|_{L} \equiv 0$.

An $m$-dimensional submanifold $L$ in $\mathbb{C}^{m}$ is called Lagrangian if $\left.\omega\right|_{L} \equiv 0$. Thus special Lagrangian submanifolds are Lagrangian submanifolds satisfying the extra condition that $\left.\operatorname{Im} \Omega\right|_{L} \equiv 0$, which is how they get their name.

\subsection{Almost Calabi-Yau $m$-folds and SL $m$-folds}

We shall define special Lagrangian submanifolds not just in Calabi-Yau manifolds, as usual, but in the much larger class of almost Calabi-Yau manifolds.

Definition 2.4 Let $m \geq 2$. An almost Calabi-Yau $m$-fold, or ACY $m$ fold for short, is a quadruple $(M, J, \omega, \Omega)$ such that $(M, J)$ is a compact $m$-dimensional complex manifold, $\omega$ is the Kähler form of a Kähler metric $g$ on $M$, and $\Omega$ is a non-vanishing holomorphic $(m, 0)$-form on $M$.

We call $(M, J, \omega, \Omega)$ a Calabi-Yau $m$-fold, or $C Y$ m-fold for short, if in addition $\omega$ and $\Omega$ satisfy

$$
\omega^{m} / m !=(-1)^{m(m-1) / 2}(i / 2)^{m} \Omega \wedge \bar{\Omega} .
$$

Then for each $x \in M$ there exists an isomorphism $T_{x} M \cong \mathbb{C}^{m}$ that identifies $g_{x}, \omega_{x}$ and $\Omega_{x}$ with the flat versions $g, \omega, \Omega$ on $\mathbb{C}^{m}$ in (2). Furthermore, $g$ is Ricci-flat and its holonomy group is a subgroup of $\mathrm{SU}(m)$.

This is not the usual definition of a Calabi-Yau manifold, but is essentially equivalent to it.

Definition 2.5 Let $(M, J, \omega, \Omega)$ be an almost Calabi-Yau $m$-fold, and $N$ an oriented real $m$-dimensional submanifold of $M$. Fix $\theta \in \mathbb{R}$. We call $N$ a special Lagrangian submanifold, or $S L m$-fold for short, with phase $\mathrm{e}^{i \theta}$ if

$$
\left.\omega\right|_{N} \equiv 0 \quad \text { and }\left.\quad(\sin \theta \operatorname{Re} \Omega-\cos \theta \operatorname{Im} \Omega)\right|_{N} \equiv 0,
$$

and $\cos \theta \operatorname{Re} \Omega+\sin \theta \operatorname{Im} \Omega$ is a positive $m$-form on the oriented $m$-fold $N$. 
Again, this is not the usual definition of special Lagrangian submanifold, but is essentially equivalent to it. Compared to Definition 2.1 we have introduced two changes:

(a) the definition now involves a phase $\mathrm{e}^{i \theta}$, and

(b) SL $m$-folds are defined by the vanishing of forms $\omega$ and $\sin \theta \operatorname{Re} \Omega-$ $\cos \theta \operatorname{Im} \Omega$, rather than as calibrated submanifolds.

The following easy lemma relates the phase of a compact SL $m$-fold to its homology class.

Lemma 2.6 Let $(M, J, \omega, \Omega)$ be an almost Calabi-Yau m-fold, and $N$ a compact $S L m$-fold in $M$ with phase $\mathrm{e}^{i \theta}$. Then

$$
[\Omega] \cdot[N]=\operatorname{Re}^{i \theta}, \quad \text { where } \quad R=\int_{N} \cos \theta \operatorname{Re} \Omega+\sin \theta \operatorname{Im} \Omega>0,
$$

$[\Omega] \in H^{m}(M, \mathbb{C})$ and $[N] \in H_{m}(M, \mathbb{Z})$. Thus the homology class $[N]$ determines the phase $\mathrm{e}^{i \theta}$ of $N$.

If we study only SL $m$-folds $N$ in a fixed homology class in $M$, then by replacing $\Omega$ by $\mathrm{e}^{-i \theta} \Omega$ we can suppose that $N$ has phase 1 , and so avoid introducing phases. However, we will later need to consider several SL 3folds $N_{1}, N_{2}, N_{3}$ in different homology classes in a Calabi-Yau 3-fold $M$, with different phases, so we can discuss what happens to SL 3-folds as we vary $[\Omega] \in H^{3}(M, \mathbb{C})$. This is why we introduced change (a).

Using the analogue of Proposition 2.3 one can show that if $(M, J, \omega, \Omega)$ is a Calabi-Yau manifold, then $N$ is special Lagrangian with phase $\mathrm{e}^{i \theta}$ if and only if it is calibrated w.r.t. $\cos \theta \operatorname{Re} \Omega+\sin \theta \operatorname{Im} \Omega$. More generally [12, §9.4], SL $m$-folds in an almost Calabi-Yau $m$-fold are calibrated w.r.t. $\cos \theta \operatorname{Re} \Omega+\sin \theta \operatorname{Im} \Omega$, but for a suitably conformally rescaled metric $g$.

Thus, we can give an equivalent definition of SL $m$-folds in terms of calibrated geometry, and change (b) above is only cosmetic. Nonetheless, in the author's view the definition of SL $m$-folds in terms of the vanishing of closed forms is more fundamental than the definition in terms of calibrated geometry, and so should be taken as the primary definition.

The deformation theory of special Lagrangian submanifolds was studied by McLean [15, §3], who proved the following result in the Calabi-Yau case with phase 1. The extension to the ACY case is described in [12, §9.5]. 
Theorem 2.7 Let $(M, J, \omega, \Omega)$ be an almost Calabi-Yau m-fold, and $N$ a compact $S L m$-fold in $M$. Then the moduli space $\mathcal{M}_{N}$ of special Lagrangian deformations of $N$ is a smooth manifold of dimension $b^{1}(N)$, the first Betti number of $N$. All elements of $\mathcal{M}_{N}$ have the same phase $\mathrm{e}^{i \theta}$, given by $[\Omega]$. $[N]=\operatorname{Re}^{i \theta}$ for $R>0$.

Using similar methods one can prove [12, §9.3, §9.5]:

Theorem 2.8 Let $\left\{\left(M, J_{t}, \omega_{t}, \Omega_{t}\right): t \in(-\epsilon, \epsilon)\right\}$ be a smooth 1-parameter family of almost Calabi-Yau m-folds. Let $N_{0}$ be a compact SL $m$-fold in $\left(M, J_{0}, \omega_{0}, \Omega_{0}\right)$, and suppose $\left[\left.\omega_{t}\right|_{N_{0}}\right]=0$ in $H^{2}\left(N_{0}, \mathbb{R}\right)$ for all $t \in(-\epsilon, \epsilon)$. Then $N_{0}$ extends to a smooth 1-parameter family $\left\{N_{t}: t \in(-\delta, \delta)\right\}$, where $0<\delta \leq \epsilon$ and $N_{t}$ is a compact $S L$ m-fold in $\left(M, J_{t}, \omega_{t}, \Omega_{t}\right)$, with phase $\mathrm{e}^{i \theta_{t}}$ determined by $\left[\Omega_{t}\right] \cdot\left[N_{0}\right]=R_{t} \mathrm{e}^{i \theta_{t}}$ for $R_{t}>0$.

Now suppose that $(M, J, \omega, \Omega)$ is an almost Calabi-Yau 3-fold, and $N$ is a special Lagrangian (rational) homology 3-sphere in $M$. Then $H^{1}(N, \mathbb{R})=$ $H^{2}(N, \mathbb{R})=0$. Thus by Theorem 2.7 the moduli space $\mathcal{M}_{N}$ has dimension 0 , so that $N$ is rigid (that is, it admits no nontrivial deformations as an SL 3 -fold in $M)$. Also, as $H^{2}(N, \mathbb{R})=0$ the condition $\left[\left.\omega_{t}\right|_{N}\right]=0$ in Theorem 2.8 holds automatically for any family of deformations $\left(J_{t}, \omega_{t}, \Omega_{t}\right)$ of the almost Calabi-Yau structure $(J, \omega, \Omega)$ on $M$. Thus, Theorem 2.8 shows that $N$ is stable under small deformations of $(J, \omega, \Omega)$, giving:

Corollary 2.9 Let $(M, J, \omega, \Omega)$ be an almost Calabi-Yau 3-fold, and $N$ a special Lagrangian homology 3-sphere in $M$. Then $N$ is rigid, and stable under small deformations of the almost Calabi-Yau structure $(J, \omega, \Omega)$ on $M$.

One moral of this is that special Lagrangian homology 3-spheres in an almost Calabi-Yau 3-fold may be a good thing to count, as they are isolated and persistent under small deformations. We will discuss this in $\S[$ ]

\section{$2.3 \quad$ Singularities of SL $m$-folds}

The author's series of papers [6, 7, 8, 9, 10, 11, 12] is mainly concerned with the study of singularities of SL $m$-folds in $\mathbb{C}^{m}$ and in almost CalabiYau manifolds. This is done both by the construction of many examples of singular SL $m$-folds in $\mathbb{C}^{m}$, and also through the development of a (partly 
conjectural) picture of how families of nonsingular SL $m$-folds can become singular, particularly when the underlying almost Calabi-Yau manifold is assumed to be generic.

We shall now briefly summarize some definitions and conjectures taken from $[12, \S 10]$. The author has sketch proofs for the conjectures (at least in complex dimension $m<6$ ) and hopes to publish full proofs fairly soon. For more details and motivation, see [12]. Here are some definitions to do with special Lagrangian cones in $\mathbb{C}^{m}$.

Definition 2.10 A (singular) SL $m$-fold $C$ in $\mathbb{C}^{m}$ is called a cone if $C=t C$ for all $t>0$, where $t C=\{t x: x \in C\}$. Let $C$ be an SL cone in $\mathbb{C}^{m}$. Then either $C$ is an $m$-plane $\mathbb{R}^{m}$ in $\mathbb{C}^{m}$, or $C$ is singular at 0 . We are interested primarily in SL cones $C$ in which 0 is the only singular point, that is, in which 0 is an isolated singularity. Then $\Sigma=C \cap \mathcal{S}^{2 m-1}$ is a compact, nonsingular $(m-1)$-submanifold of $\mathcal{S}^{2 m-1}$. We define the number of ends at infinity of $C$ to be the number $k$ of connected components of $\Sigma$.

Let $C$ be an SL cone in $\mathbb{C}^{m}$ with an isolated singularity at 0 , and let $\Sigma=C \cap \mathcal{S}^{2 m-1}$. Regard $\Sigma$ as a compact Riemannian manifold, with metric induced from the round metric in $\mathcal{S}^{2 m-1}$. Let $\Delta=\mathrm{d}^{*} \mathrm{~d}$ be the Laplacian on functions on $\Sigma$. Define the Legendrian index l-ind $(C)$ to be the number of eigenvalues of $\Delta$ in $(0,2 m)$, counted with multiplicity.

Let the connected components of $\Sigma$ be $\Sigma_{1}, \ldots, \Sigma_{k}$. Define the cone $C$ to be rigid if for each $j=1, \ldots, k$, the eigenspace of $\Delta$ on $\Sigma_{j}$ with eigenvalue $2 m$ has dimension $\operatorname{dim} S U(m)-\operatorname{dim} G_{j}$, where $G_{j}$ is the Lie subgroup of $\mathrm{SU}(m)$ preserving $\Sigma_{j}$.

The point of these definitions is that the Legendrian index of $C$ is that $\Sigma$ is a minimal Legendrian submanifold in $\mathcal{S}^{2 m-1}$, and is thus a stationary point of the area functional amongst all Legendrian submanifolds in $\mathcal{S}^{2 m-1}$. The Legendrian index is the index of this stationary point. The cone $C$ is the union of one-ended cones $C_{1}, \ldots, C_{k}$ intersecting at 0 , and $C$ is rigid if all infinitesimal deformations of $C_{j}$ as an SL cone come from infinitesimal rotations of $C_{j}$ by $\mathrm{SU}(m)$ matrices, for each $j$.

Now SL cones are important because they are local models for the simplest kind of singularities of SL $m$-folds in almost Calabi-Yau $m$-folds. To understand how singular SL $m$-folds modelled upon an SL cone $C$ in $\mathbb{C}^{m}$ can arise as limits of nonsingular SL $m$-folds, we need to consider SL $m$-folds $L$ in $\mathbb{C}^{m}$ asymptotic to $C$ at infinity. 
Definition 2.11 Let $C$ be an SL cone in $\mathbb{C}^{m}$ with isolated singularity at 0 , and let $\Sigma=C \cap \mathcal{S}^{2 m-1}$, so that $\Sigma$ is a compact, nonsingular $(m-1)$-manifold. Let $h$ be the metric on $\Sigma$ induced by the metric $g$ on $\mathbb{C}^{m}$, and $r$ the radius function on $\mathbb{C}^{m}$. Define $\iota: \Sigma \times(0, \infty) \rightarrow \mathbb{C}^{m}$ by $\iota(\sigma, r)=r \sigma$. Then the image of $\iota$ is $C \backslash\{0\}$, and $\iota^{*}(g)=r^{2} h+\mathrm{d} r^{2}$ is the cone metric on $C \backslash\{0\}$.

Let $L$ be a closed, nonsingular SL $m$-fold in $\mathbb{C}^{m}$. We call $L$ Asymptotically Conical (AC) with cone $C$ if there exists a compact subset $K \subset L$ and a diffeomorphism $\phi: \Sigma \times(R, \infty) \rightarrow L \backslash K$ for some $R>0$, such that $|\phi-\iota|=o(r)$ and $\left|\nabla^{k}(\phi-\iota)\right|=o\left(r^{1-k}\right)$ as $r \rightarrow \infty$ for $k=1,2, \ldots$, where $\nabla$ is the Levi-Civita connection of the cone metric $\iota^{*}(g)$, and $|$.$| is computed$ using $\iota^{*}(g)$.

In [12, §10] this notion of Asymptotically Conical is referred to as weakly Asymptotically Conical, to distinguish it from a second class of strongly Asymptotically Conical SL $m$-folds which converge to $C$ to order $O\left(r^{-1}\right)$ rather than $o(r)$. However, we will not need the idea of strongly AC SL $m$ folds in this paper. The following conjecture [12, Conj. 10.3] is the analogue of Theorem 2.7 for AC SL $m$-folds.

Conjecture 2.12 Let $L$ be an $A C S L m$-fold in $\mathbb{C}^{m}$, with cone $C$, and let $k$ be the number of ends of $C$ at infinity. Then the moduli space $\mathcal{M}_{L}$ of $A C S L$ $m$-folds in $\mathbb{C}^{m}$ with cone $C$ is near $L$ a smooth manifold of dimension $b^{1}(L)+$ $k-1+\operatorname{lind}(C)$.

Our next conjecture [12, Conj. 10.7] is a first approximation to the kinds of deformation results the author expects to hold for singular SL $m$-folds in almost Calabi-Yau $m$-folds.

Conjecture 2.13 Let $C$ be a rigid $S L$ cone in $\mathbb{C}^{m}$ with an isolated singularity at 0 and $k$ ends at infinity, and $L$ be an $A C S L m$-fold in $\mathbb{C}^{m}$ with cone $C$. Let $(M, J, \omega, \Omega)$ be a generic almost Calabi-Yau $m$-fold, and $\mathcal{M}$ a connected moduli space of compact nonsingular $S L m$-folds $N$ in $M$.

Suppose that at the boundary of $\mathcal{M}$ there is a moduli space $\mathcal{M}_{C}$ of compact, singular SL $m$-folds with one isolated singular point modelled on the cone $C$, which arise as limits of $S L$ m-folds in $\mathcal{M}$ by collapsing $A C S L$ folds with the topology of L. Then

$$
\operatorname{dim} \mathcal{M}=\operatorname{dim} \mathcal{M}_{C}+b^{1}(L)+k-1+\operatorname{lind}(C)-2 m .
$$


Suppose we have a suitably generic almost Calabi-Yau $m$-fold $M$ and a compact, singular SL $m$-fold $N_{0}$ in $M$, which is the limit of a family of compact nonsingular SL $m$-folds $N$ in $M$. We (loosely) define the index of the singularities of $N_{0}$ to be the codimension of the family of singular SL $m$-folds with singularities like those of $N_{0}$ in the family of nonsingular SL $m$-folds $N$. Thus, in the situation of Conjecture 2.13, the index of the singularities is $b^{1}(L)+k-1+1-i n d(C)-2 m$.

More generally, one can work not just with a fixed generic almost CalabiYau $m$-fold, but with a generic family of almost Calabi-Yau $m$-folds. So, for instance, if we have a generic $k$-dimensional family of almost Calabi-Yau $m$-folds $M$, and in each $M$ we have an $l$-dimensional family of SL $m$-folds, then in the total $(k+l)$-dimensional family of SL $m$-folds we are guaranteed to meet singularities of index at most $k+l$.

Now later in the paper we shall study the behaviour of 0-dimensional families of SL 3-folds in generic 1-dimensional families of almost Calabi-Yau 3 -folds. In such families we will only meet singularities of index 1 . This is a very useful fact, as it means there will be only a few kinds of singular behaviour to worry about in determining how the invariants we define behave under deformations of the underlying almost Calabi-Yau 3-fold.

One important reason we have chosen to work in almost Calabi-Yau manifolds, rather than just in Calabi-Yau manifolds, is that almost Calabi-Yau manifolds occur in infinite-dimensional families. Thus, taking the underlying almost Calabi-Yau manifold to be generic is a very powerful assumption, and should simplify the singular behaviour of SL $m$-folds considerably. (For instance, one can argue that a compact SL 3-fold in a generic almost CalabiYau 3-fold has at most finitely many singular points.) However, CalabiYau manifolds only occur in finite-dimensional families, and so working in a generic Calabi-Yau manifold is not that strong an assumption, and probably will not help very much.

\section{A model degeneration of SL 3-folds}

We now define an explicit SL cone $L_{0}$ in $\mathbb{C}^{3}$ and three families of AC SL 3 -folds $L_{t}^{a}$ in $\mathbb{C}^{3}$ with cone $L_{0}$, and analyze them in the framework of $\$ 2.3$. 


\subsection{Three families of SL 3 -folds in $\mathbb{C}^{3}$}

Let $G$ be the group $\mathrm{U}(1)^{2}$, acting on $\mathbb{C}^{3}$ by

$$
\left(\mathrm{e}^{i \theta_{1}}, \mathrm{e}^{i \theta_{2}}\right):\left(z_{1}, z_{2}, z_{3}\right) \mapsto\left(\mathrm{e}^{i \theta_{1}} z_{1}, \mathrm{e}^{i \theta_{2}} z_{2}, \mathrm{e}^{-i \theta_{1}-i \theta_{2}} z_{3}\right) \quad \text { for } \theta_{1}, \theta_{2} \in \mathbb{R} .
$$

All the $G$-invariant special Lagrangian 3 -folds in $\mathbb{C}^{3}$ were written down explicitly by Harvey and Lawson [5, $\S$ III.3.A], and studied in more detail in [6, Ex. 5.1] and [9, §4]. Here are some examples of $G$-invariant SL 3-folds which will be important in what follows.

Definition 3.1 Define a subset $L_{0}$ in $\mathbb{C}^{3}$ by

$$
\begin{aligned}
L_{0}=\left\{\left(z_{1}, z_{2}, z_{3}\right) \in \mathbb{C}^{3}:\left|z_{1}\right|^{2}=\left|z_{2}\right|^{2}=\left|z_{3}\right|^{2},\right. \\
\left.\operatorname{Im}\left(z_{1} z_{2} z_{3}\right)=0, \quad \operatorname{Re}\left(z_{1} z_{2} z_{3}\right) \geq 0\right\} .
\end{aligned}
$$

Then $L_{0}$ is a special Lagrangian cone on $T^{2}$, invariant under the Lie subgroup $G$ of $\mathrm{SU}(3)$ given in (17). Let $t>0$, and define

$$
\begin{aligned}
& L_{t}^{1}=\left\{\left(z_{1}, z_{2}, z_{3}\right) \in \mathbb{C}^{3}:\left|z_{1}\right|^{2}-t=\left|z_{2}\right|^{2}=\left|z_{3}\right|^{2},\right. \\
& \left.\operatorname{Im}\left(z_{1} z_{2} z_{3}\right)=0, \quad \operatorname{Re}\left(z_{1} z_{2} z_{3}\right) \geq 0\right\} . \\
& L_{t}^{2}=\left\{\left(z_{1}, z_{2}, z_{3}\right) \in \mathbb{C}^{3}:\left|z_{1}\right|^{2}=\left|z_{2}\right|^{2}-t=\left|z_{3}\right|^{2}\right. \\
& \left.\operatorname{Im}\left(z_{1} z_{2} z_{3}\right)=0, \operatorname{Re}\left(z_{1} z_{2} z_{3}\right) \geq 0\right\}, \\
& L_{t}^{3}=\left\{\left(z_{1}, z_{2}, z_{3}\right) \in \mathbb{C}^{3}:\left|z_{1}\right|^{2}=\left|z_{2}\right|^{2}=\left|z_{3}\right|^{2}-t,\right. \\
& \left.\operatorname{Im}\left(z_{1} z_{2} z_{3}\right)=0, \operatorname{Re}\left(z_{1} z_{2} z_{3}\right) \geq 0\right\} .
\end{aligned}
$$

Then it can be shown that each $L_{t}^{a}$ is a $G$-invariant, nonsingular, embedded special Lagrangian 3-submanifold in $\mathbb{C}^{3}$ diffeomorphic to $\mathcal{S}^{1} \times \mathbb{R}^{2}$, which is Asymptotically Conical in the sense of Definition 2.10, with cone $L_{0}$.

Thus the $L_{t}^{a}$ for $a=1,2,3$ are three different families of AC SL 3-folds in $\mathbb{C}^{3}$ asymptotic to the same SL cone $L_{0}$, each family depending on a real parameter $t>0$. Define subsets $D_{t}^{1}$ and $\gamma_{t}^{1}$ in $\mathbb{C}^{3}$ for $a>0$ by

$$
\gamma_{t}^{1}=\left\{\left(t^{1 / 2} \mathrm{e}^{i \theta}, 0,0\right): \theta \in \mathbb{R}\right\}, \quad D_{t}^{1}=\left\{\left(z_{1}, 0,0\right): z_{1} \in \mathbb{C}, \quad\left|z_{1}\right|^{2} \leq t\right\} .
$$

Then $\gamma_{t}^{1}$ is a smooth, oriented $\mathcal{S}^{1}$ in $L_{t}^{1}$, and $D_{t}^{1}$ is a closed, oriented holomorphic disc in $\mathbb{C}^{3}$ with area $\pi t$ and boundary $\gamma_{t}^{1}$. The homology class of $\gamma_{t}^{1}$ generates $H_{1}\left(L_{t}^{1}, \mathbb{Z}\right) \cong \mathbb{Z}$. There are similar holomorphic discs $D_{t}^{2}$ with boundary $\gamma_{t}^{2}$ in $L_{t}^{2}$, and $D_{t}^{3}$ with boundary $\gamma_{t}^{3}$ in $L_{t}^{3}$. 


\subsection{Using $L_{0}$ and the $L_{t}^{a}$ as local models}

Next we apply the ideas of $\$ 2.3$ to $L_{0}$ and the $L_{t}^{a}$.

Lemma 3.2 In the notation of $\$ 2.3$, the $S L$ cone $L_{0}$ and $A C S L 3$-folds $L_{t}^{a}$ satisfy $k=1, b^{1}\left(L_{t}^{a}\right)=1$ and $1-\operatorname{ind}\left(L_{0}\right)=6$, and $L_{0}$ is rigid.

Proof. As $L_{0}$ is a $T^{2}$-cone we have $k=1$, and as $L_{t}^{a}$ is diffeomorphic to $\mathcal{S}^{1} \times \mathbb{R}^{2}$ we have $b^{1}\left(L_{t}^{a}\right)=1$. It is not difficult to show that the metric on $\Sigma \cong T^{2}$ is isometric to the quotient of $\mathbb{R}^{2}$ with its flat Euclidean metric by the lattice $\mathbb{Z}^{2}$ with basis $2 \pi\left(\frac{\sqrt{2}}{\sqrt{3}}, 0\right), 2 \pi\left(\frac{1}{\sqrt{6}}, \frac{1}{\sqrt{2}}\right)$. The eigenvectors of $\Delta$ on $\Sigma$ lift to functions of the form $\sin (\alpha x+\beta y), \cos (\alpha x+\beta y)$ on $\mathbb{R}^{2}$ which are invariant under lattice translations.

Calculation shows that the only eigenvalue of $\Delta$ in $(0,6)$ is 2 , with multiplicity 6 , and so l-ind $\left(L_{0}\right)=6$. Similarly, $\Delta$ has eigenvalue 6 with multiplicity 6 , and as $\operatorname{dim} \mathrm{SU}(3)=8$ and the subgroup $G$ of $\mathrm{SU}(3)$ preserving $L_{0}$ is $\mathrm{U}(1)^{2}$ with dimension 2 , we see that $L_{0}$ is rigid as $6=8-2$.

Assuming Conjecture 2.12, we see that the moduli space of AC SL 3-folds $L$ with cone $L_{0}$ and $b^{1}(L)=1$ is 1-dimensional. It follows that any such $L$ must be $G$-invariant, as otherwise the $G$-orbit of $L$ and its rescalings would fill out a moduli space of larger dimension. Thus any such $L$ is one of the $L_{t}^{a}$, and we have:

Corollary 3.3 Suppose Conjecture 2.12 holds when $m=3$. Then the moduli space of $A C S L 3$-folds $L$ in $\mathbb{C}^{3}$ with cone $L_{0}$ and $b^{1}(L)=1$ is $\left\{L_{t}^{a}: a=\right.$ $1,2,3, t>0\}$.

Assuming Conjecture 2.13, we deduce:

Corollary 3.4 Suppose Conjecture 2.13 holds when $m=3$. Let $(M, J, \omega, \Omega)$ be a generic almost Calabi-Yau 3-fold, and $\mathcal{M}$ a connected moduli space of compact nonsingular SL 3-folds $N$ in $M$. Suppose that at the boundary of $\mathcal{M}$ there is a moduli space $\mathcal{M}_{0}$ of compact, singular $S L$ 3-folds with one isolated singular point modelled on the cone $L_{0}$, which arise as limits of $S L 3$-folds in $\mathcal{M}$ by collapsing $A C S L 3$-folds of the form $L_{t}^{a}$. Then $\operatorname{dim} \mathcal{M}=\operatorname{dim} \mathcal{M}_{0}+1$.

Thus, in the sense discussed in $\S 2.3$, singularities of SL 3-folds modelled on the SL cone $L_{0}$ should have index one. That is, they should occur in codimension 1 in families of SL 3-folds in a generic almost Calabi-Yau 3fold, or more generally in generic families of almost Calabi-Yau 3-folds. 


\subsection{Interpretation in terms of holomorphic discs}

Here is an informal way to understand why singularities of SL 3-folds modelled on $L_{0}$ should have index one, in terms of holomorphic discs. Given a symplectic manifold $(M, \omega)$ with compatible almost complex structure $J$, and a Lagrangian submanifold $N$ in $M$, one can consider $J$-holomorphic discs $D$ in $M$ with boundary $\partial D$ in $N$.

The behaviour of such $J$-holomorphic discs is well understood, because of their application in the Floer homology of Lagrangian submanifolds. It is known (see for instance [2, Th. 3.11]) that if $J$ and $N$ are sufficiently generic, then moduli spaces of $J$-holomorphic discs are smooth manifolds, with dimension given by a topological formula.

In our case, as $N$ is special Lagrangian the 'Maslov index' of $D$ is automatically 0 , and as $m=3$ the formula gives dimension 0 for the moduli space of holomorphic discs. The conclusion is that if $(M, J, \omega, \Omega)$ is a generic almost Calabi-Yau 3-fold and $N$ an SL 3-fold in $M$, then holomorphic discs $D$ with boundary $\partial D$ in $N$ are expected to be both isolated and stable, so that they persist under small deformations of $(J, \omega, \Omega)$ and $N$.

In the situation of Corollary 3.4, suppose that $N_{0}$ is a singular SL 3-fold in $\mathcal{M}_{0}$ with singular point $x$, and $N$ is an SL 3 -fold in $\mathcal{M}$ close to $N_{0}$. Then we expect that $N$ should be modelled near $x$ on some $L_{t}^{a}$ for small $t>0$. (This will be made more precise in $\S$ 国.) Since there is a holomorphic disc $D_{t}^{a}$ in $\mathbb{C}^{3}$ with boundary in $L_{t}^{a}$, and such discs are isolated and stable under small perturbations, we expect that there should exist a holomorphic disc $D$ in $M$ with boundary in $N$ modelled on $D_{t}^{a}$.

As $D$ is calibrated with respect to $\omega$, its area is $\int_{D} \omega$. Thus, the area of $D$ is given topologically in terms of relative homology and cohomology by the formula $\operatorname{Area}(D)=[\omega]_{M ; N} \cdot[D]_{M ; N}$, where $[\omega]_{M ; N} \in H^{2}(M ; N, \mathbb{R})$ and $[D]_{M ; N} \in H_{2}(M ; N, \mathbb{Z})$. But also, the area of $D$ is necessarily positive. When the area shrinks to zero, the boundary $\mathcal{S}^{1}$ of $D$ in $N$ is collapsed to a point, and $N$ develops a singularity, a $T^{2}$-cone.

Thus, we have the following picture. Regard $[D]_{M, N}$ as a fixed class $\beta \in H_{2}(M ; N, \mathbb{Z})$. As we continuously vary $(M, J, \omega, \Omega)$ and $N$, the class $[\omega]_{M ; N}$ varies in $H^{2}(M ; N, \mathbb{R})$. On $\mathcal{M}$ we have $[\omega]_{M ; N} \cdot \beta>0$, and $\mathcal{M}_{0}$ is the boundary where $[\omega]_{M ; N} \cdot \beta=0$. So, singularities modelled on the cone $L_{0}$ should occur at the zeroes of one topologically determined real function $[\omega]_{M ; N} \cdot \beta$ on the moduli space of SL 3 -folds, which explains why they happen in codimension one. 
The author expects that the SL cone $L_{0}$ in $\mathbb{C}^{3}$ is the generic local model for singularities of SL 3-folds which occur when the area of a holomorphic disc $D$ with boundary in an SL 3 -fold $N$ shrinks to zero.

\section{Topological behaviour of these singularities}

In $\S 3$ we argued that singularities modelled on the SL cone $L_{0}$ are of index one, in the sense of $\$ 2.3$. Therefore we expect singularities modelled on $L_{0}$ to occur in codimension one in families of SL 3-folds in generic families of almost Calabi-Yau 3-folds. We shall consider such a family, and investigate the topological consequences.

\subsection{SL 3-folds with $T^{2}$-cone singularities}

In the following condition we set out the situation we want to study, an SL 3 -fold in an almost Calabi-Yau 3-fold with one singular point modelled on $L_{0}$, and establish some notation.

Condition 4.1 Let $\left(M, J_{0}, \omega_{0}, \Omega_{0}\right)$ be an almost Calabi-Yau 3-fold, and $N_{0}$ a compact, embedded, singular SL 3 -fold in $M$ with phase $\mathrm{e}^{i \theta}$ and one singular point at $x \in M$, locally modelled on $L_{0}$. To be more precise, let $\rho>0$ be small, $B_{\rho}$ and $\bar{B}_{\rho}$ be the open and closed balls of radius $\rho$ about 0 in $\mathbb{C}^{3}$, and $\left(J^{\prime}, \omega^{\prime}, \Omega^{\prime}\right)$ the Euclidean Calabi-Yau structure on $\bar{B}_{\rho} \subset \mathbb{C}^{3}$. Suppose there exists a $C^{1}$ embedding $\Phi_{0}: \bar{B}_{\rho} \rightarrow M$ which is smooth except perhaps at 0 , such that

(a) $\Phi_{0}(0)=x$ and $\Phi_{0}^{*}\left(N_{0}\right)=L_{0} \cap \bar{B}_{\rho}$;

(b) $\Phi_{0}^{*}\left(\omega_{0}\right)=\omega^{\prime}$; and

(c) $\Phi_{0}^{*}\left(J_{0}\right) \approx J^{\prime}$ and $\Phi_{0}^{*}\left(\Omega_{0}\right) \approx F \mathrm{e}^{i \theta} \Omega^{\prime}$ near $0 \in \bar{B}_{\rho}$, for some $F>0$.

Suppose also that $H_{1}\left(N_{0}, \mathbb{R}\right)=\{0\}$.

Here are some remarks on this condition:

- As our conclusions will be conjectural, we will not worry very much about the details here - for instance, exactly what sort of approximation is required in part (c), or how many derivatives of $\Phi_{0}$ exist at $x$. 
- We have chosen the coordinate system $\Phi_{0}$ to equate the symplectic structures $\omega_{0}$ on $M$ and $\omega^{\prime}$ on $\mathbb{C}^{3}$. This is possible by the Darboux Lemma. As there are so many symplectomorphisms we can also arrange that $\left(\Phi_{0}\right)^{*}\left(N_{0}\right)=L_{0} \cap \bar{B}_{\rho}$, possibly at the cost of $\Phi_{0}$ not being smooth at 0 . However, $\Phi_{0}$ will in general not be holomorphic, so we can only assume that $\Phi_{0}^{*}\left(J_{0}\right) \approx J^{\prime}$ and $\Phi_{0}^{*}\left(\Omega_{0}\right) \approx F \mathrm{e}^{i \theta} \Omega^{\prime}$.

Here the factor $\mathrm{e}^{i \theta}$ is to compensate for the fact that $N_{0}$ has phase $\mathrm{e}^{i \theta}$ and $L_{0}$ has phase 1 , and $F$ is to allow for the fact that as $\left(M, J_{0}, \omega_{0}, \Omega_{0}\right)$ is only an almost Calabi-Yau 3-fold, so $\left(J_{0}, \omega_{0}, \Omega_{0}\right)$ is not necessarily isomorphic to $\left(J^{\prime}, \omega^{\prime}, \Omega^{\prime}\right)$ at $x$, but only to $\left(J^{\prime}, \omega^{\prime}, F \Omega^{\prime}\right)$ for some $F>0$.

One could instead choose the $\Phi_{0}$ to be holomorphic coordinates, with $\Phi_{0}^{*}\left(J_{0}\right)=J^{\prime}$ and $\Phi_{0}^{*}\left(\Omega_{0}\right)=\Omega^{\prime}$. But then the best we could hope for would be $\Phi_{0}^{*}\left(\omega_{0}\right) \approx F \omega^{\prime}$ and $\Phi_{0}^{*}\left(N_{0}\right) \approx L_{0} \cap \bar{B}_{\rho}$.

- The assumptions that $N_{0}$ is embedded and $H_{1}\left(N_{0}, \mathbb{R}\right)=\{0\}$ are to simplify the calculations involving (relative) homology and cohomology below, and because we will eventually only be interested in special Lagrangian homology 3-spheres $N$, which have $H_{1}(N, \mathbb{R})=\{0\}$. The assumptions can be removed without great difficulty.

Suppose Condition 4.1 holds. Define $P=N_{0} \backslash \Phi_{0}\left(B_{\rho}\right)$. Then $P$ is a compact, nonsingular 3-manifold whose boundary is the image under $\Phi_{0}$ of the intersection of $L_{0}$ with the sphere $\mathcal{S}_{\rho}^{5}$ in $\mathbb{C}^{3}$ of radius $\rho$. Now $L_{0} \cap \mathcal{S}_{\rho}^{5}$ is the orbit of $3^{-1 / 2} \rho(1,1,1)$ under the action of $G=\mathrm{U}(1)^{2}$ defined in (7). So define $\iota: G \rightarrow P$ by

$$
\iota:\left(\mathrm{e}^{i \theta_{1}}, \mathrm{e}^{i \theta_{2}}\right) \mapsto \Phi_{0}\left(\frac{1}{\sqrt{3}} \rho \mathrm{e}^{i \theta_{1}}, \frac{1}{\sqrt{3}} \rho \mathrm{e}^{i \theta_{2}}, \frac{1}{\sqrt{3}} \rho \mathrm{e}^{-i \theta_{1}-i \theta_{2}}\right) .
$$

Then $\iota$ is a diffeomorphism $G \rightarrow \partial P$.

Lemma 4.2 The inclusion $\iota: G \rightarrow P$ induces a map $\iota_{*}: H_{1}(G, \mathbb{Z}) \rightarrow$ $H_{1}(P, \mathbb{Z})$, which has $\operatorname{Ker}\left(\iota_{*}\right) \cong \mathbb{Z}$.

Proof. As $H_{1}(G, \mathbb{Z}) \cong \mathbb{Z}^{2}$ and $\operatorname{Ker}\left(\iota_{*}\right)$ is a subgroup of $H_{1}(G, \mathbb{Z})$, we see that $\operatorname{Ker}\left(\iota_{*}\right)$ is isomorphic to $0, \mathbb{Z}$ or $\mathbb{Z}^{2}$. But using Poincaré duality ideas for manifolds with boundary, we can show that the map $H_{1}(G, \mathbb{R}) \rightarrow H_{1}(P, \mathbb{R})$ must have image and kernel $\mathbb{R}$, and this forces $\operatorname{Ker}\left(\iota_{*}\right) \cong \mathbb{Z}$. 
Using Poincaré duality for $P$ and $H_{1}\left(N_{0}, \mathbb{R}\right)=\{0\}$ one can also show that $H_{1}(P, \mathbb{R}) \cong \mathbb{R}$ and $H_{2}(P, \mathbb{R})=\{0\}$. Write $Q_{0}=N_{0} \cap \Phi_{0}\left(\bar{B}_{\rho}\right)$. Then $Q_{0}$ is a singular 3-manifold with boundary $\partial Q_{0}=\partial P=\iota(G)$. Topologically $Q_{0}$ is the cone on $\partial P$, and is contractible. We shall now define homology classes $\zeta, \chi$ and integers $k^{1}, k^{2}, k^{3}$ which will be important in what follows.

Definition 4.3 Let $\zeta \in H_{1}(G, \mathbb{Z})$ be a generator for $\operatorname{Ker}\left(\iota_{*}\right) \cong \mathbb{Z}$. Then $\zeta$ is unique up to sign. Now $G=\mathrm{U}(1)^{2}$, so we may identify $H_{1}(G, \mathbb{Z}) \cong \mathbb{Z}^{2}$ in the obvious way, such that the maps $\mathcal{S}^{1} \rightarrow G$ given by $\mathrm{e}^{i \theta} \mapsto\left(\mathrm{e}^{i \theta}, 1\right)$ and $\mathrm{e}^{i \theta} \mapsto\left(1, \mathrm{e}^{i \theta}\right)$ represent the classes in $H_{1}(G, \mathbb{Z})$ identified with $(1,0)$ and $(0,1)$ in $\mathbb{Z}^{2}$ respectively. Define $k^{1}, k^{2}, k^{3} \in \mathbb{Z}$ so that $\zeta \in H_{1}(G, \mathbb{Z})$ is identified with $\left(k^{1}, k^{2}\right) \in \mathbb{Z}^{2}$, and $k^{3}=-k^{1}-k^{2}$. Then $k^{1}+k^{2}+k^{3}=0$.

Let $\tau$ be a closed integral 1-chain in $\partial P$ with $[\tau]=\iota_{*}(\zeta)$ in $H_{1}(\partial P, \mathbb{Z})$. Then $[\tau]=\iota_{*}(\zeta)=0$ in $H_{1}(P, \mathbb{Z})$, so there exists an integral 2-chain $\Lambda$ in $P$ with $\partial \Lambda=\tau$. Also $\tau$ is a closed integral 1-chain in $\partial Q_{0}$ and $Q_{0}$ is contractible, so there exists an integral 2-chain $\Sigma$ in $Q_{0}$ with $\partial \Sigma=\tau$. Thus $\Sigma-\Lambda$ is an integral 2-chain without boundary in $N_{0} \subset M$. Define $\chi=$ $[\Sigma-\Lambda] \in H_{2}(M, \mathbb{R})$. Then $\chi$ is a homology class in the image of $H_{2}(M, \mathbb{Z})$ in $H_{2}(M, \mathbb{R})$. Since $H_{2}(P, \mathbb{R})=H_{2}\left(Q_{0}, \mathbb{R}\right)=\{0\}$ this $\chi$ is independent of the choice of $\Lambda, \Sigma$, and so is unique up to the choice of sign of $\zeta$.

Note that as the chain $\Sigma-\Lambda$ representing $\chi$ lies in $N_{0}$, which is Lagrangian with respect to $\omega_{0}$, we have $\left[\omega_{0}\right] \cdot \chi=0$, where $\left[\omega_{0}\right] \in H^{2}(M, \mathbb{R})$ is the Kähler class of $\omega_{0}$. In the rest of the section we shall consider separately the cases $\chi \neq 0$ and $\chi=0$.

\subsection{The case $\chi \neq 0$ : desingularizing $N_{0}$}

Let $\left(M, J_{0}, \omega_{0}, \Omega_{0}\right), N_{0}$ and $x$ be as above. We shall now consider the question of when $N_{0}$ is the limit as $t \rightarrow 0_{+}$of a family of compact, nonsingular SL 3 -folds modelled on $L_{t}^{a}$ near $x$. In general this cannot happen in the fixed almost Calabi-Yau 3-fold $\left(M, J_{0}, \omega_{0}, \Omega_{0}\right)$. Instead we need to suppose $\chi \neq 0$, and extend $\left(M, J_{0}, \omega_{0}, \Omega_{0}\right)$ to a smooth family of almost Calabi-Yau 3-folds $\left(M, J_{t}, \omega_{t}, \Omega_{t}\right)$ with $\left[\omega_{t}\right] \cdot \chi=t$. Here is our conjecture.

Conjecture 4.4 Let Condition 4.1 hold, and $k^{1}, k^{2}, k^{3}$ and $\chi$ be as in Definition 4.3. Suppose $\chi \neq 0$, and $\left(M, J_{0}, \omega_{0}, \Omega_{0}\right)$ extends to a smooth family $\left\{\left(M, J_{t}, \omega_{t}, \Omega_{t}\right): t \in(-\epsilon, \epsilon)\right\}$ of almost Calabi-Yau 3-folds for some $\epsilon>0$, where $\left[\omega_{t}\right] \cdot \chi=t$. 
Then there do not exist singular SL 3 -folds $N_{t}$ in $\left(M, J_{t}, \omega_{t}, \Omega_{t}\right)$ close to $N_{0}$ and of the same topological type for $t \neq 0$. However, for some small $\delta \in(0, \epsilon]$, whenever $a=1,2,3,|t|<\delta$ and $k^{a} t>0$ there exists a compact, nonsingular, embedded $S L$ 3-fold $N_{t}^{a}$ in $\left(M, J_{t}, \omega_{t}, \Omega_{t}\right)$ with phase $\mathrm{e}^{i \theta_{t}}$, which depends smoothly on $t$, and converges to $N_{0}$ as $t \rightarrow 0$ in a suitable sense, and near $x$ is locally modelled on $L_{t / k^{a} \pi}^{a}$.

To be more precise, whenever $a=1,2,3,0<|t|<\delta$ and $k^{a} t>0$, there exists an embedding $\Phi_{t}^{a}: \bar{B}_{\rho} \rightarrow M$ which depends smoothly on $t$ and converges to $\Phi_{0}$ as $t \rightarrow 0$, and satisfies

(a) $\left(\Phi_{t}^{a}\right)^{*}\left(N_{t}^{a}\right)=L_{t / k^{a} \pi}^{a} \cap \bar{B}_{\rho}$;

(b) $\left(\Phi_{t}^{a}\right)^{*}\left(\omega_{t}\right)=\omega^{\prime}$; and

(c) $\left(\Phi_{t}^{a}\right)^{*}\left(J_{t}\right) \approx J^{\prime}$ and $\left(\Phi_{t}^{a}\right)^{*}\left(\Omega_{t}\right) \approx F_{t} \mathrm{e}^{i \theta_{t}} \Omega^{\prime}$ for small $t$, near $0 \in \bar{B}_{\rho}$, for some $F_{t}>0$.

In the rest of this subsection we shall give a partial proof of this conjecture, which justifies the assertion that $N_{t}$ does not exist for $t \neq 0$ but that $N_{t}^{a}$ should exist when $k^{a} t>0$, and be locally modelled on $L_{t / k^{a} \pi}^{a}$, rather than $L_{s}^{a}$ for some other $s>0$. To complete the proof will require essentially the same analysis needed to solve Conjecture 2.13, and so once Conjecture 2.13 has been proved, completing the conjecture above should be straightforward.

First, the nonexistence of singular SL 3-folds $N_{t}$ in $\left(M, J_{t}, \omega_{t}, \Omega_{t}\right)$ for $t \neq 0$ close to $N_{0}$, and of the same topological type. Suppose such an $N_{t}$ did exist. Then $N_{t}$ is homeomorphic to $N_{0}$, and as in $\S 4.1$ we can construct a closed integral 2-chain $C$ in $N_{t}$ close to $\Sigma-\Lambda$ in $N_{0}$. Then $[C]=\chi$ in the image of $H_{2}(M, \mathbb{Z})$ in $H_{2}(M, \mathbb{R})$, as $\chi$ is a discrete object and is unchanged under small variations. But $\left.\omega_{t}\right|_{C}=0$, as $C$ lies in $N_{t}$ which is Lagrangian with respect to $\omega_{t}$. Hence $\left[\omega_{t}\right] \cdot \chi=0$, which contradicts the assumptions $\left[\omega_{t}\right] \cdot \chi=t$ and $t \neq 0$.

Suppose that for some small $t \in(-\epsilon, \epsilon)$ there exists a compact nonsingular SL 3-fold $N_{t}^{a}$ in $\left(M, J_{t}, \omega_{t}, \Omega_{t}\right)$, which is close to $N_{0}$ in a suitable sense, and near $x$ is modelled on $L_{s}^{a}$. That is, there should exist an embedding $\Phi_{t}^{a}$ : $\bar{B}_{\rho} \rightarrow M$ satisfying $\left(\Phi_{t}^{a}\right)^{*}\left(N_{t}^{a}\right)=L_{s}^{a} \cap \bar{B}_{\rho}$ and parts (b), (c) above.

Define $P_{t}^{a}=N_{t}^{a} \backslash \Phi_{t}^{a}\left(B_{\rho}\right)$ and $Q_{t}^{a}=N_{t}^{a} \cup \Phi_{t}^{a}\left(\bar{B}_{\rho}\right)$. Then $Q_{t}^{a}$ is the image under $\Phi_{t}^{a}$ of $L_{s}^{a} \cap \bar{B}_{\rho}$. Provided $s<\rho^{2}$, which we may assume as $s$ is small, $Q_{t}^{a}$ is diffeomorphic to $\mathcal{S}^{1} \times D^{2}$, and $P_{t}^{a}$ is a compact, nonsingular 3-manifold 
with boundary $\partial P_{t}^{a}=\partial Q_{t}^{a} \cong T^{2}$. Furthermore, $P_{t}^{a}$ is diffeomorphic to $P$ in $\$ 4.1$. (In our present situation this follows from the assumption that $N_{t}^{a}$ is close to $N_{0}$, and in the situation of the conjecture from the fact that $P_{t}^{a}$ depends smoothly on $t$ and converges to $P$ as $t \rightarrow 0$.)

Now the boundary of $L_{s}^{a} \cap \bar{B}_{\rho}$ is a $G$-orbit, so by picking a point in the boundary and using $\Phi_{t}^{a}$ we may define a diffeomorphism $\iota_{t}^{a}: G \rightarrow \partial P_{t}^{a}=\partial Q_{t}^{a}$, in the same way that we defined $\iota$ in $\S 4.1$. Furthermore, the diffeomorphism $P \cong P_{t}^{a}$ may be chosen to identify $\iota$ and $\iota_{t}^{a}$.

Let $\zeta$ be as in Definition 4.3, and let $\tau_{t}^{a}$ be a closed integral 1-chain in $\partial P_{t}^{a}$ with $\left[\tau_{t}^{a}\right]=\left(\iota_{t}^{a}\right)_{*}(\zeta)$ in $H_{1}\left(\partial P_{t}^{a}, \mathbb{Z}\right)$. Then $\left[\tau_{t}^{a}\right]=\left(\iota_{t}^{a}\right)_{*}(\zeta)=0$ in $H_{1}\left(P_{t}^{a}, \mathbb{Z}\right)$, so there exists an integral 2-chain $\Lambda_{t}^{a}$ in $P_{t}^{a}$ with $\partial \Lambda_{t}^{a}=\tau_{t}^{a}$. In 33 we defined a holomorphic disc $D_{s}^{a}$ in $\mathbb{C}^{3}$ with boundary $\partial D_{s}^{a}=\gamma_{s}^{a}$ in $L_{s}^{a}$. Thus $\Phi_{s}^{a}\left(\gamma_{s}^{a}\right)$ is an oriented $\mathcal{S}^{1}$ in $Q_{t}^{a}$. As $Q_{t}^{a} \cong \mathcal{S}^{1} \times D^{2}$ we have $H_{1}\left(Q_{t}^{a}, \mathbb{Z}\right) \cong \mathbb{Z}$, and it is easy to see that $\left[\Phi_{s}^{a}\left(\gamma_{s}^{a}\right)\right]$ generates $H_{1}\left(Q_{t}^{a}, \mathbb{Z}\right)$.

Now $\tau_{t}^{a}$ is a closed 1-chain in $\partial Q_{t}^{a}$, and the integers $k^{1}, k^{2}, k^{3}$ of Definition 4.3 were chosen to ensure that $\left[\tau_{t}^{a}\right]=\left(\iota_{t}^{a}\right)_{*}(\zeta)=k^{a}\left[\Phi_{s}^{a}\left(\gamma_{s}^{a}\right)\right]$ in $H_{1}\left(Q_{s}^{a}, \mathbb{Z}\right)$. Therefore $\tau_{t}^{a}$ is homologous to $k^{a} \Phi_{t}^{a}\left(\gamma_{s}^{a}\right)$ in $Q_{t}^{a}$, so there exists an integral 2-chain $\Sigma_{t}^{a}$ in $Q_{t}^{a}$ with $\partial \Sigma_{t}^{a}=\tau_{t}^{a}-k^{a} \Phi_{t}^{a}\left(\gamma_{s}^{a}\right)$.

Consider the integral 2-chain $k^{a} \Phi_{t}^{a}\left(D_{s}^{a}\right)+\Sigma_{t}^{a}-\Lambda_{t}^{a}$ in $M$. As $\partial D_{s}^{a}=\gamma_{s}^{a}$, $\partial \Sigma_{t}^{a}=\tau_{t}^{a}-k^{a} \Phi_{t}^{a}\left(\gamma_{s}^{a}\right)$ and $\partial \Lambda_{t}^{a}=\tau_{t}^{a}$ it is closed, and so defines a homology class in the image of $H_{2}(M, \mathbb{Z})$ in $H_{2}(M, \mathbb{R})$. Now this class is exactly $\chi$, as we constructed it by a small deformation of the construction of $\chi$ in Definition 4.3, but $\chi$ is a discrete object and so is unchanged by continuous deformations. Therefore we have

$$
\left[\omega_{t}\right] \cdot \chi=k^{a} \int_{\Phi_{t}^{a}\left(D_{s}^{a}\right)} \omega_{t}+\int_{\Sigma_{t}^{a}} \omega_{t}-\int_{\Lambda_{t}^{a}} \omega_{t}=k^{a} \int_{D_{s}^{a}} \omega^{\prime}=k^{a} \pi s
$$

using part (b) of the conjecture and the fact that $\Sigma_{t}^{a}$ and $\Lambda_{t}^{a}$ lie in $N_{t}^{a}$, which is Lagrangian with respect to $\omega_{t}$.

Now $\left[\omega_{t}\right] \cdot \chi=t$ by definition, so we have shown that $t=k^{a} \pi s$. But $s>0$, as $L_{s}^{a}$ is only defined for positive $s$. Thus, if $t$ and $k^{a}$ are not both zero then $N_{t}^{a}$ can only exist if $k^{a} t>0$, and then $s=t / k^{a} \pi$, as we have to prove. The case $t=k^{a}=0$ is excluded by the conjecture, and will be discussed in $\$ 4.3$. This concludes our partial proof of Conjecture 4.4. 


\subsection{The case $\chi \neq 0$ : homology 3 -spheres}

Next we prove that the 3 -manifolds $N_{t}^{a}$ above are homology 3-spheres, and calculate the size of $H_{1}\left(N_{t}^{a}, \mathbb{Z}\right)$.

Proposition 4.5 In the situation of Conjecture 4.4 , both $H_{1}\left(N_{0}, \mathbb{Z}\right)$ and $H_{1}\left(N_{t}^{a}, \mathbb{Z}\right)$ are finite and satisfy $\left|H_{1}\left(N_{t}^{a}, \mathbb{Z}\right)\right|=\left|k^{a}\right| \cdot\left|H_{1}\left(N_{0}, \mathbb{Z}\right)\right|$. Thus, each $N_{t}^{a}$ is a (rational) homology 3-sphere.

Proof. Let $a=1,2$ or 3 and $t$ satisfy $|t|<\delta$ and $k^{a} t>0$, and let $N_{t}^{a}$ be as in Conjecture 4.4. Suppose also that $t$ is small enough that $|t|<\left|k^{a}\right| \pi \rho^{2}$, to ensure that $Q_{t}^{a} \cong \mathcal{S}^{1} \times D^{2}$, and define $P_{t}^{a}, Q_{t}^{a}$ and $\iota_{t}^{a}$ as in $\$ 4.2$. As the $N_{t}^{a}$ depend smoothly on $t$ they are all diffeomorphic for fixed $a$, and so it is enough to prove the proposition for sufficiently small $t$.

Using the exact sequence

$$
H_{1}(\{x\}, \mathbb{Z}) \rightarrow H_{1}\left(N_{0}, \mathbb{Z}\right) \rightarrow H_{1}\left(N_{0} ;\{x\}, \mathbb{Z}\right) \rightarrow H_{0}(\{x\}, \mathbb{Z}) \stackrel{\cong}{\longrightarrow} H_{0}\left(N_{0}, \mathbb{Z}\right)
$$

and $H_{1}(\{x\}, \mathbb{Z})=0$, we find that $H_{1}\left(N_{0}, \mathbb{Z}\right) \cong H_{1}\left(N_{0} ;\{x\}, \mathbb{Z}\right)$, and by excision and the diffeomorphism $P \cong P_{t}^{a}$ we see that $H_{1}\left(N_{0} ;\{x\}, \mathbb{Z}\right) \cong$ $H_{1}\left(P_{t}^{a} ; \partial P_{t}^{a}, \mathbb{Z}\right) \cong H_{1}\left(N_{t}^{a} ; Q_{t}^{a}, \mathbb{Z}\right)$. Thus $H_{1}\left(N_{t}^{a} ; Q_{t}^{a}, \mathbb{Z}\right) \cong H_{1}\left(N_{0}, \mathbb{Z}\right)$. Recall that $H_{1}\left(N_{0}, \mathbb{R}\right)=\{0\}$ by Condition 4.1. It is easy to show using this and facts about manifold topology that $H_{1}\left(N_{0}, \mathbb{Z}\right)$ is finite.

As $Q_{t}^{a}$ is diffeomorphic to $\mathcal{S}^{1} \times \mathbb{R}^{2}$ we see that $H_{1}\left(Q_{t}^{a}, \mathbb{Z}\right)$ is isomorphic to $\mathbb{Z}$, and is generated by $\left[\Phi_{t}^{a}\left(\gamma_{t / k^{a} \pi}^{a}\right)\right]$. But the argument in $\$ 4.2$ shows that $k^{a} \Phi_{t}^{a}\left(\gamma_{t / k^{a} \pi}^{a}\right)$ is homologous in $Q_{t}^{a}$ to $\tau_{t}^{a}$ in $\partial Q_{t}^{a}$, and $\tau_{t}^{a}$ is homologous to 0 in $P_{t}^{a}$. Thus, the image of $\left|k^{a}\right|\left[\Phi_{t}^{a}\left(\gamma_{t / k^{a} \pi}^{a}\right)\right]$ in $H_{1}\left(N_{t}^{a}, \mathbb{Z}\right)$ is zero. Furthermore, no smaller positive multiple of $\left[\Phi_{t}^{a}\left(\gamma_{t / k^{a} \pi}^{a}\right)\right]$ can have zero image in $H_{1}\left(N_{t}^{a}, \mathbb{Z}\right)$, because $\tau_{t}^{a}$ represents $\zeta$, which by definition generates the kernel of $\left(\iota_{t}^{a}\right)_{*}$.

Now consider the exact sequence

$$
H_{1}\left(Q_{t}^{a}, \mathbb{Z}\right) \rightarrow H_{1}\left(N_{t}^{a}, \mathbb{Z}\right) \rightarrow H_{1}\left(N_{t}^{a} ; Q_{t}^{a}, \mathbb{Z}\right) \rightarrow H_{0}\left(Q_{t}^{a}, \mathbb{Z}\right) \stackrel{\cong}{\longrightarrow} H_{0}\left(N_{t}^{a}, \mathbb{Z}\right) .
$$

We have shown above that $H_{1}\left(N_{t}^{a} ; Q_{t}^{a}, \mathbb{Z}\right) \cong H_{1}\left(N_{0}, \mathbb{Z}\right)$, which is finite, and that the image of $H_{1}\left(Q_{t}^{a}, \mathbb{Z}\right)$ in $H_{1}\left(N_{t}^{a}, \mathbb{Z}\right)$ is isomorphic to the cyclic group $\mathbb{Z}_{\left|k^{a}\right|}$. Thus by exactness $H_{1}\left(N_{t}^{a}, \mathbb{Z}\right) / \mathbb{Z}_{\left|k^{a}\right|} \cong H_{1}\left(N_{0}, \mathbb{Z}\right)$, and so $H_{1}\left(N_{t}^{a}, \mathbb{Z}\right)$ is finite with $\left|H_{1}\left(N_{t}^{a}, \mathbb{Z}\right)\right|=\left|k^{a}\right| \cdot\left|H_{1}\left(N_{0}, \mathbb{Z}\right)\right|$, as we have to prove. 
Since $H_{1}\left(N_{t}^{a}, \mathbb{Z}\right)$ is finite we have $b_{1}\left(N_{t}^{a}\right)=0$, so $b_{2}\left(N_{t}^{a}\right)=0$ by Poincaré duality. But $N_{t}^{a}$ is connected (this is part of our definition of manifold) and oriented, as it is special Lagrangian. So $N_{t}^{a}$ is by definition a rational homology 3-sphere.

Our goal in this paper is to define an invariant of almost Calabi-Yau 3folds by counting special Lagrangian homology 3 -spheres in some appropriate way. We can use the ideas above to draw some conclusions about how to do this. We assume for the moment that Conjecture 4.4 is true.

Because $k^{1}+k^{2}+k^{3}=0$ and the $k^{a}$ are not all zero as $\zeta \neq 0$, at least one $k^{a}$ is positive, and one negative. But the SL 3 -fold $N_{t}^{a}$ exists for small $t>0$ if and only if $k^{a}>0$, and for small $t<0$ if and only if $k^{a}<0$. So, consider the following three cases:

(a) Suppose $k_{1}, k_{2}>0$ and $k_{3}<0$. Then $N_{t}^{1}$ and $N_{t}^{2}$ exist as SL 3-folds for small $t>0$ but $N_{t}^{3}$ does not, and $N_{t}^{3}$ exists for small $t<0$ but $N_{t}^{1}, N_{t}^{2}$ do not.

(b) Suppose $k_{1}>0, k_{2}=0$ and $k_{3}<0$. Then $N_{t}^{1}$ exists for small $t>0$, $N_{t}^{3}$ exists for small $t<0$, and $N_{t}^{2}$ does not exist for any $t \neq 0$.

(c) Suppose $k_{1}>0$ and $k_{2}, k_{3}<0$. Then $N_{t}^{1}$ exists for small $t>0$, and $N_{t}^{2}, N_{t}^{3}$ exist for small $t<0$.

These show that as we deform $\left(M, J_{t}, \omega_{t}, \Omega_{t}\right)$, changing the Kähler class $\left[\omega_{t}\right]$, it can happen that two SL homology 3-spheres disappear, and one reappears; or that one disappears, and another reappears; or that one disappears, and two reappear.

In particular, this shows that the number of special Lagrangian homology 3 -spheres in $M$ is not invariant under deformations of $(M, J, \omega, \Omega)$ changing the Kähler class $[\omega]$, even if counted with signs. So, simple counting of SL homology 3 -spheres, even with signs, is probably not the right thing to do. However, it is easy to see using Proposition 4.5 that the sum over SL homology 3-spheres $N$ of the weight $\left|H_{1}(N, \mathbb{Z})\right|$ is unchanged under transitions of the kind described in Conjecture 1.4 .

For instance, in case (a) above, for small $t>0$ there exist two SL homology 3-spheres $N_{t}^{1}, N_{t}^{2}$, with $\left|H_{1}\left(N_{t}^{1}, \mathbb{Z}\right)\right|=k^{1}\left|H_{1}\left(N_{0}, \mathbb{Z}\right)\right|$ and $\left|H_{1}\left(N_{t}^{2}, \mathbb{Z}\right)\right|=$ $k^{2}\left|H_{1}\left(N_{0}, \mathbb{Z}\right)\right|$, and when $t<0$ there is one SL homology 3-spheres $N_{t}^{3}$ with $\left|H_{1}\left(N_{t}^{3}, \mathbb{Z}\right)\right|=-k^{3}\left|H_{1}\left(N_{0}, \mathbb{Z}\right)\right|$. But as $-k^{3}=k^{1}+k^{2}$ we see that $\left|H_{1}\left(N_{t}^{1}, \mathbb{Z}\right)\right|+\left|H_{1}\left(N_{t}^{2}, \mathbb{Z}\right)\right|=\left|H_{1}\left(N_{t}^{3}, \mathbb{Z}\right)\right|$, so the sum of weights is the same 
for small $t>0$ and $t<0$. This suggests that the appropriate thing to do is to count SL homology 3 -spheres $N$ with weight $\left|H_{1}(N, \mathbb{Z})\right|$, and we will argue this in $\oint \rrbracket$.

We can now discuss the existence of SL 3-folds $N_{t}^{a}$ when $k^{a}=t=0$, which was passed over in $\$ 4.2$. The argument of $\$ 4.2$ shows that for an SL 3 -fold $N_{t}^{a}$ to exist in $\left(M, J_{t}, \omega_{t}, \Omega_{t}\right)$ that is modelled on $N_{0}$ away from $x$ and $L_{s}^{a}$ near $x$, we need $t=k^{a} \pi s$. Thus, if $k^{a}=0$ then such SL 3-folds can only exist when $t=0$. The argument above gives an exact sequence

$$
\mathbb{Z}=H_{1}\left(Q_{t}^{a}, \mathbb{Z}\right) \rightarrow H_{1}\left(N_{t}^{a}, \mathbb{Z}\right) \rightarrow H_{1}\left(N_{0}, \mathbb{Z}\right) \rightarrow 0
$$

and shows that the generator of $H_{1}\left(Q_{t}^{a}, \mathbb{Z}\right)$ has order $\left|k^{a}\right|$ in $H_{1}\left(N_{t}^{a}, \mathbb{Z}\right)$. However, in this case $k^{a}=0$, so the $\operatorname{map} \mathbb{Z} \rightarrow H_{1}\left(N_{t}^{a}, \mathbb{Z}\right)$ is injective. It follows that $H_{1}\left(N_{t}^{a}, \mathbb{Z}\right)$ is infinite, the product of $\mathbb{Z}$ with a finite group, and hence that $b^{1}\left(N_{t}^{a}\right)=1$. So $N_{t}^{a}$ is not a homology 3-sphere, and by Theorem 2.7 it is not isolated as an SL 3-fold, but occurs in a moduli space of dimension 1.

Thus we are led to the following picture. If $k^{a}>0$ then there exists a unique SL 3-fold $N_{t}^{a}$ in $\left(M, J_{t}, \omega_{t}, \Omega_{t}\right)$ for small $t>0$. If $k^{a}<0$ then there exists a unique SL 3 -fold $N_{t}^{a}$ in $\left(M, J_{t}, \omega_{t}, \Omega_{t}\right)$ for small $-t>0$. And if $k^{a}=0$ then for each small $s>0$ there exists a unique SL 3 -fold $N_{0}^{a}$ in $\left(M, J_{0}, \omega_{0}, \Omega_{0}\right)$ modelled near $x$ on $L_{s}^{a}$. So in each case the SL 3-folds are indexed by a small positive parameter.

\subsection{The case $\chi=0$ : stable SL singularities}

In $\S 3.2$ we argued that SL singularities modelled on the SL cone $L_{0}$ in $\mathbb{C}^{3}$ are of index one, and should occur in codimension one in families of SL 3-folds in generic families of almost Calabi-Yau 3-folds. However, such local calculations of the 'index' of singularities can sometimes give the wrong answer in cases when global topological restrictions come into play. This happens when $\chi=0$ in the situation of $\$ 4.1$, and effectively the index of the singularities should be zero rather than one in this case.

Let $\left\{\left(M, J_{t}, \omega_{t}, \Omega_{t}\right): t \in(-\epsilon, \epsilon)\right\}$ be a smooth family of almost CalabiYau 3-folds deforming $\left(M, J_{0}, \omega_{0}, \Omega_{0}\right)$. As $\chi=0$, we cannot assume that $\left[\omega_{t}\right] \cdot \chi=t$, so the family is essentially arbitrary. The argument in $\$ 4.2$ that there do not exists singular SL 3 -folds $N_{t}$ in $\left(M, J_{t}, \omega_{t}, \Omega_{t}\right)$ for $t \neq 0$ close to $N_{0}$, and of the same topological type, is now no longer valid, and the author conjectures that such $N_{t}$ do exist for small $t$. 
What about the existence of nonsingular SL 3-folds resolving $N_{0}$ ? The argument in $\$ 4.2$ shows that if for small $t$ there exists a nonsingular SL 3-fold $N_{s, t}^{a}$ in $\left(M, J_{t}, \omega_{t}, \Omega_{t}\right)$ close to $N_{0}$ away from $x$ and modelled on $L_{s}^{a}$ near $x$, then $k^{a} \pi s=\left[\omega_{t}\right] \cdot \chi=0$. As $s>0$, this shows that such SL 3-folds $N_{s, t}^{a}$ cannot exist unless $k^{a}=0$. When $k^{a}=0$ such $N_{s, t}^{a}$ can exist, and have $b^{1}\left(N_{s, t}^{a}\right)=1$ as in $\$ 4.3$, so they should occur in 1-parameter families in $\left(M, J_{t}, \omega_{t}, \Omega_{t}\right)$ by Theorem 2.7, parametrized by $s$.

We summarize our conclusions in the following conjecture, which should be provable by the same methods as Conjecture 4.4.

Conjecture 4.6 Let Condition 4.1 hold, and $k^{1}, k^{2}, k^{3}$ and $\chi$ be as in Definition 4.3. Suppose $\chi=0$, and $\left(M, J_{0}, \omega_{0}, \Omega_{0}\right)$ extends to a smooth family $\left\{\left(M, J_{t}, \omega_{t}, \Omega_{t}\right): t \in(-\epsilon, \epsilon)\right\}$ of almost Calabi-Yau 3-folds for some $\epsilon>0$. Then for some $\delta \in(0, \epsilon], N_{0}$ extends to a smooth family of compact, embedded, singular $S L$ 3-folds $N_{t}$ in $\left(M, J_{t}, \omega_{t}, \Omega_{t}\right)$ for $t \in(-\delta, \delta)$, each of which has one singular point locally modelled on $L_{0}$.

If $k^{a}=0$ then for small $t \in(-\epsilon, \epsilon)$ and $s>0$ there exists a unique compact, nonsingular $S L 3$-fold $N_{s, t}^{a}$ in $\left(M, J_{t}, \omega_{t}, \Omega_{t}\right)$ close to $N_{0}$ away from $x$, and modelled on $L_{s}^{a}$ near $x$. If $k^{a} \neq 0$ there do not exist any such $N_{s, t}^{a}$.

\section{Another model degeneration of SL 3-folds}

We now describe a family of explicit SL 3-folds $K_{\phi, A}$ in $\mathbb{C}^{3}$. This family was first found by Lawlor [13], was made more explicit by Harvey [4, p. 139-140], and was discussed from a different point of view by the author in [0, §5.4(b)]. Our treatment is based on that of Harvey.

Let $a_{1}, a_{2}, a_{3}>0$, and define polynomials $p(x), P(x)$ by

$$
p(x)=\left(1+a_{1} x^{2}\right)\left(1+a_{2} x^{2}\right)\left(1+a_{3} x^{2}\right)-1 \quad \text { and } \quad P(x)=\frac{p(x)}{x^{2}} .
$$

Define real numbers $\phi_{1}, \phi_{2}, \phi_{3}$ and $A$ by

$$
\phi_{k}=a_{k} \int_{-\infty}^{\infty} \frac{\mathrm{d} x}{\left(1+a_{k} x^{2}\right) \sqrt{P(x)}} \quad \text { and } \quad A=\frac{4 \pi}{3}\left(a_{1} a_{2} a_{3}\right)^{-1 / 2} .
$$

Clearly $\phi_{k}>0$ and $A>0$. But writing $\phi_{1}+\phi_{2}+\phi_{3}$ as one integral and rearranging gives

$$
\phi_{1}+\phi_{2}+\phi_{3}=\int_{0}^{\infty} \frac{p^{\prime}(x) \mathrm{d} x}{(p(x)+1) \sqrt{p(x)}}=2 \int_{0}^{\infty} \frac{\mathrm{d} w}{w^{2}+1}=\pi,
$$


making the substitution $w=\sqrt{p(x)}$. So $\phi_{k} \in(0, \pi)$ and $\phi_{1}+\phi_{2}+\phi_{3}=\pi$. It can be shown that this yields a 1-1 correspondence between triples $\left(a_{1}, a_{2}, a_{3}\right)$ with $a_{k}>0$, and quadruples $\left(\phi_{1}, \phi_{2}, \phi_{3}, A\right)$ with $\phi_{k} \in(0, \pi), \phi_{1}+\phi_{2}+\phi_{3}=\pi$ and $A>0$.

For $k=1,2,3$ and $y \in \mathbb{R}$, define $z_{k}(y)$ by $z_{k}(y)=\mathrm{e}^{i \psi_{k}(y)} \sqrt{a_{k}^{-1}+y^{2}}$, where

$$
\psi_{k}(y)=a_{k} \int_{-\infty}^{y} \frac{\mathrm{d} x}{\left(1+a_{k} x^{2}\right) \sqrt{P(x)}} .
$$

Now write $\boldsymbol{\phi}=\left(\phi_{1}, \phi_{2}, \phi_{3}\right)$, and define a submanifold $K_{\phi, A}$ in $\mathbb{C}^{3}$ by

$$
K_{\boldsymbol{\phi}, A}=\left\{\left(z_{1}(y) x_{1}, z_{2}(y) x_{2}, z_{3}(y) x_{3}\right): y \in \mathbb{R}, x_{k} \in \mathbb{R}, x_{1}^{2}+x_{2}^{2}+x_{3}^{2}=1\right\} \text {. }
$$

Our next result comes from Harvey [凹, Th. 7.78].

Proposition 5.1 The set $K_{\phi, A}$ defined in (14) is an embedded SL 3-fold in $\mathbb{C}^{3}$ diffeomorphic to $\mathcal{S}^{2} \times \mathbb{R}$. It is asymptotically conical, with cone the union $\Pi_{0} \cup \Pi_{\phi}$ of two special Lagrangian 3-planes $\Pi_{0}, \Pi_{\phi}$ given by

$$
\Pi_{0}=\left\{\left(x_{1}, x_{2}, x_{3}\right): x_{j} \in \mathbb{R}\right\}, \Pi_{\phi}=\left\{\left(\mathrm{e}^{i \phi_{1}} x_{1}, \mathrm{e}^{i \phi_{2}} x_{2}, \mathrm{e}^{i \phi_{3}} x_{3}\right): x_{j} \in \mathbb{R}\right\} \text {. }
$$

Here is how to interpret the constant $A$. Using the above notation, define

$$
D_{\phi, A}=\left\{\left(x_{1} \mathrm{e}^{i \phi_{1} / 2}, x_{2} \mathrm{e}^{i \phi_{2} / 2}, x_{3} \mathrm{e}^{i \phi_{3} / 2}\right): x_{k} \in \mathbb{R}, a_{1} x_{1}^{2}+a_{2} x_{2}^{2}+a_{3} x_{3}^{2} \leq 1\right\} .
$$

Then $D_{\phi, A}$ is a solid ellipsoid in $\mathbb{C}^{3}$, with boundary in $K_{\phi, A}$. The axes of $D_{\phi, A}$ have lengths $a_{k}^{-1 / 2}$ for $k=1,2,3$, and so the volume of $D_{\phi, A}$ is $A$. Furthermore, $D_{\phi, A}$ is calibrated with respect to $\operatorname{Im}\left(\Omega_{0}\right)$. That is, we can regard $D_{\phi, A}$ as an SL 3 -fold of phase $i$, whereas $K_{\phi, A}$ has phase 1 ; so that $D_{\phi, A}$ and $K_{\phi, A}$ are both special Lagrangian, but of perpendicular phase.

We met a similar situation in $\$$. 3 . There we defined an AC SL 3-fold $L_{t}^{a}$ depending on a real parameter $t>0$, and a holomorphic 2-disc $D_{t}^{a}$ with boundary on $L_{t}^{a}$, and area $\pi t$. Here we define an AC SL 3 -fold $K_{\phi, A}$ depending on a real parameter $A>0$, and a special Lagrangian 3 -disc $D_{\phi, A}$ of phase $i$, with boundary on $K_{\phi, A}$ and area $A$.

Next we apply the ideas of $\S 2.3$ to $\Pi_{0} \cup \Pi_{\boldsymbol{\phi}}$ and the $K_{\boldsymbol{\phi}, A}$. 
Lemma 5.2 In the notation of $\S .3, \Pi_{0} \cup \Pi_{\phi}$ and $K_{\phi, A}$ satisfy $k=2$, $b^{1}\left(K_{\phi, A}\right)=0$ and $1-\operatorname{ind}\left(K_{\phi, A}\right)=6$, and $\Pi_{0} \cup \Pi_{\phi}$ is rigid.

Proof. As $\Pi_{0} \cup \Pi_{\phi}$ is a cone on two disjoint copies of $\mathcal{S}^{2}$ we have $k=1$, and as $K_{\phi, A}$ is diffeomorphic to $\mathcal{S}^{2} \times \mathbb{R}$ we have $b^{1}\left(K_{\phi, A}\right)=0$. There are two connected components $\Sigma_{1}, \Sigma_{2}$ of $\Sigma$, each of which is isometric to the unit sphere $\mathcal{S}^{2}$ in $\mathbb{R}^{3}$ with the round metric.

For both $\Sigma_{1}, \Sigma_{2}$ the only eigenvalue of $\Delta$ in $(0,6)$ is 2 , with multiplicity 3 , and eigenvectors the restriction to $\mathcal{S}^{2}$ of linear functions on $\mathbb{R}^{3}$. So l-ind $\left(K_{\phi, A}\right)=3+3=6$. Similarly, $\Delta$ has eigenvalue 6 with multiplicity 5 on each of $\Sigma_{1}, \Sigma_{2}$, and eigenfunctions the restrictions to $\mathcal{S}^{2}$ of harmonic homogeneous quadratic polynomials on $\mathbb{R}^{3}$. As $\operatorname{dim} \mathrm{SU}(3)=8$ and the subgroup $G_{j}$ of $\mathrm{SU}(3)$ preserving $\Sigma_{j}$ is isomorphic to $\mathrm{SO}(3)$ with dimension 3 , we see that $\Pi_{0} \cup \Pi_{\phi}$ is rigid as $5=8-3$.

Assuming Conjecture 2.13, we see that in the sense discussed in $\$ 2.3$, singularities of SL 3-folds modelled on the SL cones $\Pi_{0} \cup \Pi_{\phi}$ should have index one. That is, they should occur in codimension 1 in families of SL 3 -folds in a generic almost Calabi-Yau 3-fold, or more generally in generic families of almost Calabi-Yau 3-folds.

Now $\Pi_{0} \cup \Pi_{\phi}$ may be regarded as a singular SL cone in $\mathbb{C}^{3}$ with isolated singular point at 0 , but it is also the union of two nonsingular special Lagrangian 3-planes. In the same way, an embedded singular SL 3-fold $N$ in $(M, J, \omega, \Omega)$ with one singular point $x$ modelled on $\Pi_{0} \cup \Pi_{\phi}$ is either a nonsingular immersed SL 3-fold with one self-intersection point at $x$, or the union of two nonsingular SL 3-folds $N^{+}, N^{-}$which intersect at $x$, and have the same phase. We shall study this second possibility.

\section{Topological behaviour of these singularities}

We shall study the following situation.

Condition 6.1 Let $\epsilon>0$, and $\left\{\left(M, J_{t}, \omega_{t}, \Omega_{t}\right): t \in(-\epsilon, \epsilon)\right\}$ be a smooth family of almost Calabi-Yau 3-folds with the same underlying 6-manifold $M$. Suppose that $N_{0}^{+}$and $N_{0}^{-}$are compact, embedded, nonsingular special Lagrangian homology 3 -spheres in $\left(M, J_{0}, \omega_{0}, \Omega_{0}\right)$ with the same phase $\mathrm{e}^{i \theta}$, which intersect transversely at one point $x$, such that the intersection $N_{0}^{+} \cap$ $N_{0}^{-}$is positive in the sense of homology, using the natural orientations on $N_{0}^{ \pm}$and $M$. 
We can show using the results of $\$ 2.2$ that $N_{0}^{ \pm}$extend to families of SL 3 -folds $N_{t}^{ \pm}$in $\left(M, J_{t}, \omega_{t}, \Omega_{t}\right)$.

Proposition 6.2 Suppose Condition 6.1 holds. Then there exists $\delta \in(0, \epsilon]$ such that $N_{0}^{+}$and $N_{0}^{-}$extend to unique smooth families $\left\{N_{t}^{ \pm}: t \in(-\delta, \delta)\right\}$ of compact, nonsingular, embedded SL 3-folds in $\left(M, J_{t}, \omega_{t}, \Omega_{t}\right)$. Each $N_{t}^{ \pm}$is diffeomorphic to $N_{0}^{ \pm}$and homologous to it in $H_{3}(M, \mathbb{Z})$, and $N_{t}^{+}$and $N_{t}^{-}$ intersect transversely in one point, where the intersection $N_{t}^{+} \cap N_{t}^{-}$is positive in the sense of homology.

Proof. As $N_{0}^{ \pm}$are homology 3-spheres we have $H^{2}\left(N_{0}^{ \pm}, \mathbb{R}\right)=0$, so the condition $\left[\left.\omega_{t}\right|_{N_{0}^{ \pm}}\right]=0$ in $H^{2}\left(N_{0}^{ \pm}, \mathbb{R}\right)$ in Theorem 2.8 holds automatically. Thus, it follows from Theorem 2.8 that for some $\delta \in(0, \epsilon]$ we can extend $N_{0}^{ \pm}$to smooth families $N_{t}^{ \pm}$of compact, nonsingular, embedded SL 3-folds in $\left(M, J_{t}, \omega_{t}, \Omega_{t}\right)$ for $t \in(-\delta, \delta)$. As these are smooth, connected families the $N_{t}^{ \pm}$are clearly diffeomorphic and homologous to $N_{0}^{ \pm}$.

Now $b^{1}\left(N_{t}^{ \pm}\right)=b^{1}\left(N_{0}^{ \pm}\right)=0$ as $N_{0}^{ \pm}$is a homology 3-sphere, so $N_{t}^{ \pm}$is rigid by Theorem 2.7, that is, it has no special Lagrangian deformations. Thus the families $N_{t}^{ \pm}$are unique. To intersect transversely in one point is an open condition on pairs of submanifolds, so by making $\delta$ smaller if necessary we can suppose that $N_{t}^{+}$and $N_{t}^{-}$intersect transversely in one point, which will be a positive intersection as $\left[N_{t}^{+}\right] \cap\left[N_{t}^{-}\right]=\left[N_{0}^{+}\right] \cap\left[N_{0}^{-}\right]=1$.

We shall use the following notation.

Definition 6.3 Let Condition 6.1 hold, and use the notation of Proposition 6.2. Define $\chi^{ \pm}=\left[N_{0}^{ \pm}\right]$in $H_{3}(M, \mathbb{Z})$. Then $\left[N_{t}^{ \pm}\right]=\chi^{ \pm}$for $t \in(-\delta, \delta)$. Let the phase of $N_{t}^{ \pm}$be $\mathrm{e}^{i \theta_{t}^{ \pm}}$. Then $\theta_{t}^{ \pm}$is only defined modulo $2 \pi \mathbb{Z}$, but we fix it uniquely by requiring that $\theta_{0}^{ \pm}=\theta$ and $\theta_{t}^{ \pm}$should depend smoothly on $t$. Applying Lemma 2.6 to $N_{t}^{ \pm}$we see that

$$
\left[\Omega_{t}\right] \cdot \chi^{ \pm}=R_{t}^{ \pm} \mathrm{e}^{i \theta_{t}^{ \pm}} \quad \text { for } t \in(-\delta, \delta),
$$

for some $R_{t}^{ \pm}>0$ depending smoothly on $t$. As $\theta_{0}^{+}=\theta_{0}^{-}=\theta$, by making $\delta>0$ smaller if necessary we may assume that

$$
\left|\theta_{t}^{+}-\theta_{t}^{-}\right|<\pi \quad \text { for all } t \in(-\delta, \delta) .
$$

Then $R_{t}^{+} \mathrm{e}^{i \theta_{t}^{+}}+R_{t}^{-} \mathrm{e}^{i \theta_{t}^{-}} \neq 0$ for any $t \in(-\delta, \delta)$. Define $R_{t}>0$ and $\theta_{t}$ by

$$
\left[\Omega_{t}\right] \cdot\left(\chi^{+}+\chi^{-}\right)=R_{t}^{+} \mathrm{e}^{i \theta_{t}^{+}}+R_{t}^{-} \mathrm{e}^{i \theta_{t}^{-}}=R_{t} \mathrm{e}^{i \theta_{t}}
$$


for all $t \in(-\delta, \delta)$. Here $\theta_{t}$ is only defined modulo $2 \pi \mathbb{Z}$, but we specify $\theta_{t}$ uniquely by requiring that it depend smoothly on $t$, and $\theta_{0}=\theta$. It is then easy to show that $\theta_{t}$ lies between $\theta_{t}^{+}$and $\theta_{t}^{-}$for all $t \in(-\delta, \delta)$.

One can show using a 'simultaneous diagonalization' argument that if $\Pi^{+}, \Pi^{-}$are SL 3-planes with phase 1 in $\mathbb{C}^{3}$ intersecting only at 0 then there exists $B \in \mathrm{SU}(3)$ such that $B \Pi^{+}=\Pi_{0}$ and $B \Pi^{-}=\Pi_{\phi}$, where

$$
\Pi_{0}=\left\{\left(x_{1}, x_{2}, x_{3}\right): x_{j} \in \mathbb{R}\right\} \quad \text { and } \quad \Pi_{\phi}=\left\{\left(\mathrm{e}^{i \phi_{1}} x_{1}, \mathrm{e}^{i \phi_{2}} x_{2}, \mathrm{e}^{i \phi_{3}} x_{3}\right): x_{j} \in \mathbb{R}\right\}
$$

for some $\phi_{1}, \phi_{2}, \phi_{3} \in(0, \pi)$, where $\phi_{1}+\phi_{2}+\phi_{3}=\pi$ if $\Pi^{+} \cap \Pi^{-}$is a positive intersection in the sense of homology, and $\phi_{1}+\phi_{2}+\phi_{3}=2 \pi$ if $\Pi^{+} \cap \Pi^{-}$is a negative intersection. Using this it is easy to prove:

Proposition 6.4 Suppose Condition 6.1 holds. Then there exists a complex linear isometry $\iota: \mathbb{C}^{3} \rightarrow T_{x} M$ satisfying

$$
\iota^{*}\left(\Omega_{0}\right)=\mathrm{e}^{i \theta} \mathrm{d} z_{1} \wedge \mathrm{d} z_{2} \wedge \mathrm{d} z_{3}, \quad \iota^{*}\left(T_{x} N_{0}^{+}\right)=\Pi_{0} \quad \text { and } \quad \iota^{*}\left(T_{x} N_{0}^{-}\right)=\Pi_{\phi},
$$

where $\phi_{1}, \phi_{2}, \phi_{3} \in(0, \pi)$ with $\phi_{1}+\phi_{2}+\phi_{3}=\pi$, and $\Pi_{0}, \Pi_{\phi}$ are given in (15).

This shows that after adjusting the phase by $\mathrm{e}^{i \theta}$, the singular SL 3-fold $N_{0}^{+} \cup N_{0}^{-}$is modelled on the SL cone $\Pi_{0} \cup \Pi_{\phi}$ in $\mathbb{C}^{3}$ considered in $\S 5$. Thus, it is natural to consider whether there exist compact, nonsingular SL 3-folds $N_{t}$ in $\left(M, J_{t}, \omega_{t}, \Omega_{t}\right)$ modelled on $N_{0}^{+} \cup N_{0}^{-}$away from $x$, and on $K_{\phi, A}$ near $x$ for small $t \in(-\delta, \delta)$ and $A>0$. Here is our conjectural answer to this question.

Conjecture 6.5 In the situation of Condition 6.1, with the notation defined above, for each small $t \in(-\delta, \delta)$ with $\theta_{t}^{+}>\theta_{t}^{-}$there exists a unique compact, nonsingular special Lagrangian 3-fold $N_{t}$ in $\left(M, J_{t}, \omega_{t}, \Omega_{t}\right)$ modelled on $N_{0}^{+} \cup$ $N_{0}^{-}$away from $x$, and on $K_{\phi, A}$ near $x$, where $A>0$ is small, depends on $t$, and is given approximately by

$$
A \approx R_{t}^{+} \sin \left(\theta_{t}^{+}-\theta_{t}\right)=R_{t}^{-} \sin \left(\theta_{t}-\theta_{t}^{-}\right) .
$$

Furthermore, $N_{t}$ is diffeomorphic to the connected sum $N_{0}^{+} \# N_{0}^{-}$and is also a homology 3-sphere, with $\left|H_{1}\left(N_{t}, \mathbb{Z}\right)\right|=\left|H_{1}\left(N_{t}^{+}, \mathbb{Z}\right)\right| \cdot\left|H_{1}\left(N_{t}^{-}, \mathbb{Z}\right)\right|$, and $\left[N_{t}\right]=\chi^{+}+\chi^{-} \in H_{3}(M, \mathbb{Z})$. If $t$ is small and $\theta_{t}^{+} \leq \theta_{t}^{-}$then there do not exist any such $S L$ 3-folds $N_{t}$. 
In the rest of the section we will give a partial proof of this conjecture, justifying the assertion that $N_{t}$ should exist only if $\theta_{t}^{+}>\theta_{t}^{-}$, the approximate value of $A$ in (21), and the topological claims about $N_{t}$. We begin with the topology. If the SL 3-fold $N_{t}$ exists then it is modelled on $N_{0}^{+} \cup N_{0}^{-}$away from $x$, and on $K_{\phi, A}$ near $x$. But $K_{\phi, A}$ is a narrow 'neck' diffeomorphic to $\mathcal{S}^{2} \times \mathbb{R}$.

Thus, topologically $N_{t}$ is made by removing the point $x$ from $N_{0}^{+}$and $N_{0}^{-}$, and joining them together with a small $\mathcal{S}^{2} \times \mathbb{R}$ 'neck'. That is, $N_{t}$ is the connected sum $N_{0}^{+} \# N_{0}^{-}$of $N_{0}^{+}$and $N_{0}^{-}$. It follows that $N_{t}$ is a homology 3 -sphere, and $H_{1}\left(N_{t}, \mathbb{Z}\right) \cong H_{1}\left(N_{t}^{+}, \mathbb{Z}\right) \times H_{1}\left(N_{t}^{-}, \mathbb{Z}\right)$, so that $\left|H_{1}\left(N_{t}, \mathbb{Z}\right)\right|=$ $\left|H_{1}\left(N_{t}^{+}, \mathbb{Z}\right)\right| \cdot\left|H_{1}\left(N_{t}^{-}, \mathbb{Z}\right)\right|$.

Therefore $b^{1}\left(N_{t}\right)=0$, and hence by Theorem $2.7 N_{t}$ is rigid, so that if it exists it should be unique. (This is not a rigorous argument). It is also obvious that $N_{t}$ is homologous to $N_{0}^{+} \cup N_{0}^{-}$, and so $\left[N_{t}\right]=\left[N_{0}^{+}\right]+\left[N_{0}^{-}\right]=$ $\chi^{+}+\chi^{-}$in $H_{3}(M, \mathbb{Z})$. Equation (19) then gives $\left[\omega_{t}\right] \cdot\left[N_{t}\right]=R_{t} \mathrm{e}^{i \theta_{t}}$. So by Lemma 2.6 the phase of $N_{t}$ is $\mathrm{e}^{i \theta_{t}}$.

Now if $N_{t}$ has phase $\mathrm{e}^{i \theta_{t}}$ and is modelled on $K_{\phi, A}$ near $x$, in a similar way to Conjecture 4.4 we expect that for some small $\rho>0$ there should exist local coordinates $\Phi_{t}: \bar{B}_{\rho} \rightarrow M$ near $x$ satisfying the conditions

(a) $\Phi_{t}^{*}\left(N_{t}\right) \approx K_{\phi, A} \cap \bar{B}_{\rho}$;

(b) $\Phi_{t}^{*}\left(\omega_{t}\right) \approx F_{t} \omega^{\prime}$ near $0 \in \bar{B}_{\rho}$ for some $F_{t}>0$; and

(c) $\Phi_{t}^{*}\left(J_{t}\right)=J^{\prime}$ and $\Phi_{t}^{*}\left(\Omega_{t}\right)=\mathrm{e}^{i \theta_{t}} \Omega^{\prime}$.

Here we have chosen our coordinate system to be holomorphic and to identify $\Omega_{t}$ with $\mathrm{e}^{i \theta_{t}} \Omega^{\prime}$, which means we can only assume that $\phi_{t}^{*}\left(N_{t}\right) \approx K_{\phi, A}$ and $\Phi_{t}^{*}\left(\omega_{t}\right) \approx F_{t} \omega^{\prime}$.

Equation (16) defined a special Lagrangian ellipsoid $D_{\phi, A}$ in $\mathbb{C}^{3}$ with phase $i$ and boundary in $K_{\phi, A}$. As $\Phi_{t}^{*}\left(N_{t}\right) \approx K_{\phi, A} \cap \bar{B}_{\rho}$ there exists a disc $D_{t}$ in $\mathbb{C}^{3}$ close to $D_{\phi, A}$ with $\partial D_{t} \subset \Phi_{t}^{*}\left(N_{t}\right)$. As $D_{t}$ is close to $D_{\phi, A}$ we have

$$
\int_{D_{t}} \operatorname{Im} \Omega^{\prime} \approx \int_{D_{\phi, A}} \operatorname{Im} \Omega^{\prime}=A,
$$

as $D_{\phi, A}$ is calibrated with respect to $\operatorname{Im} \Omega^{\prime}$ and has area $A$.

Now $N_{t} \backslash \Phi_{t}\left(\partial D_{t}\right)$ has two connected components, say $P_{t}^{ \pm}$, where $P_{t}^{+}$ is close to $N_{0}^{+}$and $P_{t}^{-}$to $N_{0}^{-}$. The closures $\bar{P}_{t}^{ \pm}$are compact, oriented 3manifolds with boundary. Careful consideration of the orientations of $N_{t}$ 
and $D_{t}$ shows that as 3-chains in $M$ we have

$$
\partial\left(\bar{P}_{t}^{+}\right)=\Phi_{t}\left(\partial D_{t}\right) \quad \text { and } \quad \partial\left(\bar{P}_{t}^{-}\right)=-\Phi_{t}\left(\partial D_{t}\right) .
$$

Therefore $\bar{P}_{t}^{ \pm} \pm \Phi_{t}\left(D_{t}\right)$ is an integral 3-chain in $M$ without boundary, and so defines a homology class in $H_{3}(M, \mathbb{Z})$. But clearly $\bar{P}_{t}^{ \pm} \pm \Phi_{t}\left(D_{t}\right)$ is homologous to $N_{0}^{ \pm}$, and so $\left[\bar{P}_{t}^{ \pm} \pm \Phi_{t}\left(D_{t}\right)\right]=\chi^{ \pm}$.

Consider the integral of the 3 -form $\operatorname{Im}\left(\mathrm{e}^{-i \theta_{t}} \Omega_{t}\right)$ over $\bar{P}_{t}^{ \pm} \pm \Phi_{t}\left(D_{t}\right)$. Since $N_{t}$ is special Lagrangian of phase $\mathrm{e}^{i \theta_{t}}$, this form vanishes on $N_{t}$ and $P_{t}^{ \pm}$. Thus

$$
\operatorname{Im}\left(\mathrm{e}^{i \theta_{t}}\left[\Omega_{t}\right] \cdot \chi^{ \pm}\right)= \pm \int_{\Phi_{t}\left(D_{t}\right)} \operatorname{Im}\left(\mathrm{e}^{-i \theta_{t}} \Omega_{t}\right)= \pm \int_{D_{t}} \operatorname{Im}\left(\Omega^{\prime}\right) \approx \pm A,
$$

using part (c) above and equation (22). Therefore

$$
A \approx \operatorname{Im}\left(\mathrm{e}^{-i \theta_{t}}\left[\Omega_{t}\right] \cdot \chi^{+}\right)=-\operatorname{Im}\left(\mathrm{e}^{-i \theta_{t}}\left[\Omega_{t}\right] \cdot \chi^{-}\right) .
$$

Substituting in equation (17) yields (21), as we have to prove.

Now $\left|\theta_{t}^{+}-\theta_{t}^{-}\right|<\pi$ by (18), and $\theta_{t}$ lies in between $\theta_{t}^{+}$and $\theta_{t}^{-}$. Using these one can show that if $\theta_{t}^{+}>\theta_{t}^{-}$then $\theta_{t}^{+}-\theta_{t}$ and $\theta_{t}-\theta_{t}^{-}$lie in $(0, \pi)$ and the (approximate) value for $A$ in (21) is positive, but if $\theta_{t}^{+} \leq \theta_{t}^{-}$then $\theta_{t}^{+}-\theta_{t}$ and $\theta_{t}-\theta_{t}^{-}$lie in $(-\pi, 0]$ and the (approximate) value for $A$ is nonpositive. Thus, $\theta_{t}^{+}>\theta_{t}^{-}$corresponds to the condition that $A>0$, which is part of the definition of $K_{\phi, A}$, and justifies our claim that $\theta_{t}^{+}>\theta_{t}^{-}$should be the necessary and sufficient condition for the existence of $N_{t}$ for small $t$. This completes our partial proof of Conjecture 6.5.

Note that Adrian Butscher [1] has proved an analytic result closely related to Conjecture 6.5, but for SL $m$-folds in $\mathbb{C}^{m}$ satisfying certain boundary conditions rather than for compact SL $m$-folds in almost Calabi-Yau $m$-folds. It seems likely that his analysis can be extended to prove our conjecture.

\section{Counting SL homology 3 -spheres}

The Gromov-Witten invariants of a symplectic manifold $(M, \omega)$ are defined by counting, with signs, the $J$-holomorphic curves in $M$ satisfying certain homological conditions. A good introduction to the subject is given by McDuff and Salamon [14]. One important feature of these invariants is that they are very stable under deformations of the choice of almost complex structure $J$ used to define them. 
Now it seems a natural (but perhaps optimistic) question to ask whether we can define similar invariants of almost Calabi-Yau 3-folds $(M, J, \omega, \Omega)$ by counting SL 3-folds $N$ in $M$ satisfying suitable homological conditions. Probably the simplest such condition is to count 3-folds $N$ in some fixed homology class in $H_{3}(N, \mathbb{Z})$, and we will focus on this. We shall also restrict our attention to (rational) homology 3 -spheres, to get zero-dimensional moduli spaces.

Thus we aim to define an invariant as follows. Let $(M, J, \omega, \Omega)$ be a almost Calabi-Yau 3-fold, let $\delta \in H_{3}(M, \mathbb{Z})$, and let $S(\delta)$ be the set of special Lagrangian homology 3 -spheres $N$ in $M$ with $[N]=\delta$. Suppose $S(\delta)$ is finite, and define

$$
I(\delta)=\sum_{N \in S(\delta)} w(N),
$$

where $w$ is a weight function taking values in a commutative ring $R$, and $w(N)$ depends only on the topology of $N$. In this way we define a map $I: H_{3}(M, \mathbb{Z}) \rightarrow R$, which we consider to be an analogue of the GromovWitten invariants. For this invariant to be interesting, we would like it to be stable under deformations of the underlying almost Calabi-Yau 3-fold $(M, J, \omega, \Omega)$, or at least to change in a predictable way as we make these deformations.

Therefore we need to know what can happen to special Lagrangian homology 3-spheres as we deform $(M, J, \omega, \Omega)$, and especially how they can become singular, appear or disappear. Each such transition may change the set of special Lagrangian homology 3-spheres, and thus the invariant $I(\delta)$. For $I(\delta)$ to be invariant or to transform nicely under these transitions, the weight function $w$ must satisfy some topological identities.

We have already described models for two such transitions in $\$$ 田 and $\S 6$. We will calculate the conditions on $w$ for $I(\delta)$ to be invariant under the change described in $\delta$, and to transform in a certain simple way under the change described in $\S 6$. It turns out that the weight function $w(N)=\left|H_{1}(N, \mathbb{Z})\right|$ satisfies both of these conditions.

We also propose corrections to (23) to include multiple covers of special Lagrangian homology 3-spheres and special Lagrangian 3-folds with stable singularities. We summarize our conclusions in Conjecture 7.3, and then discuss the connections between our invariants and String Theory. 


\subsection{Invariance of $I(\delta)$ under the transitions of 3 田}

In $\$ 4.2-\$ 4.3$ we explained how, as we deform an almost Calabi-Yau 3-fold $\left(M, J_{t}, \omega_{t}, \Omega_{t}\right)$ in a 1-parameter family, three SL 3 -folds $N_{t}^{1}, N_{t}^{2}, N_{t}^{3}$ can converge to the same singular SL 3 -fold $N_{0}$ with a $T^{2}$-cone singularity. This happens on a hyperplane $\left[\omega_{t}\right] \cdot \chi=0$ in the Kähler cone, and $N_{t}^{a}$ exists as a nonsingular SL 3-fold in $(M, J, \omega, \Omega)$ if either $k^{a}>0$ and $\left[\omega_{t}\right] \cdot \chi>0$, or $k^{a}<0$ and $\left[\omega_{t}\right] \cdot \chi<0$. It is easy to see that the condition for the invariant $I(\delta)$ given by $(23)$ to be unchanged by this transition is

$$
\sum_{a \in\{1,2,3\}: k^{a}>0} w\left(N_{t}^{a}\right)=\sum_{a \in\{1,2,3\}: k^{a}<0} w\left(N_{t}^{a}\right) .
$$

Now if $N$ is a homology 3-sphere then $H_{1}(N, \mathbb{Z})$ is a finite group, so $\left|H_{1}(N, \mathbb{Z})\right|$ is a positive integer. Take the commutative ring $R$ to be $\mathbb{Z}$, and define $w(N)=\left|H_{1}(N, \mathbb{Z})\right|$. Remarkably, it turns out that this weight function, perhaps the simplest nontrivial invariant of $N$ there is, satisfies (24).

Proposition 7.1 Define an integer-valued invariant $w$ of compact, nonsingular 3-manifolds $N$ by $w(N)=\left|H_{1}(N, \mathbb{Z})\right|$ if $H_{1}(N, \mathbb{Z})$ is finite, and $w(N)=0$ if $H_{1}(N, \mathbb{Z})$ is infinite. Then (24) holds for all sets of 3-manifolds $N_{t}^{1}, N_{t}^{2}, N_{t}^{3}$ constructed as in $\$$. 9 .

The proof follows quickly from the material of $\S$.

\subsection{Transformation of $I(\delta)$ under the transitions of $\S 6$}

We shall use the following notation.

Definition 7.2 Let $M$ be a compact 6-manifold, and suppose $\chi^{+}, \chi^{-} \in$ $H_{3}(M, \mathbb{Z})$ are linearly independent over $\mathbb{R}$. Define a subset $W\left(\chi^{+}, \chi^{-}\right)$in $H^{3}(M, \mathbb{C})$ by

$$
W\left(\chi^{+}, \chi^{-}\right)=\left\{\Phi \in H^{3}(M, \mathbb{C}):\left(\Phi \cdot \chi^{+}\right)\left(\bar{\Phi} \cdot \chi^{-}\right) \in(0, \infty)\right\} .
$$

Then $W\left(\chi^{+}, \chi^{-}\right)$is a real hypersurface in $H^{3}(M, \mathbb{C})$, but not a hyperplane. Let $\Phi \in H^{3}(M, \mathbb{C})$, and write $\left(\Phi \cdot \chi^{+}\right)\left(\bar{\Phi} \cdot \chi^{-}\right)=R \mathrm{e}^{i \theta}$, where $R \geq 0$ and $\theta \in(-\pi, \pi]$. Then $\Phi \in W\left(\chi^{+}, \chi^{-}\right)$if $R>0$ and $\theta=0$. Let $\epsilon>0$ be small. We say that $\Phi$ lies on the positive side of $W\left(\chi^{+}, \chi^{-}\right)$if $R>0$ and $\theta \in(0, \epsilon)$, and on the negative side of $W\left(\chi^{+}, \chi^{-}\right)$if $R>0$ and $\theta \in(-\epsilon, 0)$. 
Section 6 studied a family of almost Calabi-Yau 3-folds $\left(M, J_{t}, \omega_{t}, \Omega_{t}\right)$ containing SL homology 3-spheres $N_{t}^{ \pm}$, with $\left[N_{t}^{ \pm}\right]=\chi^{ \pm}$in $H_{3}(M, \mathbb{Z})$, such that $N_{t}^{+} \cup N_{t}^{-}$is a transverse intersection at a single point, and positive in homology. In the notation above, the phases $\mathrm{e}^{i \theta_{t}^{ \pm}}$of $N_{t}^{ \pm}$are equal exactly when $\left[\Omega_{t}\right] \in W\left(\chi^{+}, \chi^{-}\right)$, and Conjecture 6.1 says that as $\left[\Omega_{t}\right]$ passes through $W\left(\chi^{+}, \chi^{-}\right)$from the negative to the positive side, a new SL 3-fold $N_{t}$ diffeomorphic to $N_{t}^{+} \# N_{t}^{-}$is created, with $\left[N_{t}\right]=\chi^{+}+\chi^{-}$.

Clearly, $N_{t}$ contributes $w\left(N_{t}\right) \neq 0$ to the invariant $I\left(\chi^{+}+\chi^{-}\right)$defined in (23). Therefore, $I\left(\chi^{+}+\chi^{-}\right)$will take different values on the positive and negative sides of $W\left(\chi^{+}, \chi^{-}\right)$. So, $I(\delta)$ will not be unchanged under deformations of the almost Calabi-Yau structure $(J, \omega, \Omega)$ on $M$ which change $[\Omega]$ in $H^{3}(M, \mathbb{C})$. Instead, we hope to arrange for $I(\delta)$ to transform according to some rules involving $[\Omega]$ and the values $I(\alpha)$ for other $\alpha$ in $H_{3}(M, \mathbb{Z})$.

To see what the simplest of these rules should be, let us generalize the situation of $\delta$, and suppose that $\left[\Omega_{t}\right]$ lies on the negative side of $W\left(\chi^{+}, \chi^{-}\right)$ for $t \in(-\delta, 0)$, on $W\left(\chi^{+}, \chi^{-}\right)$for $t=0$, and on the positive side of $W\left(\chi^{+}, \chi^{-}\right)$ for $t \in(0, \delta)$, but that $N_{0}^{+}$and $N_{0}^{-}$intersect transversely at $k+l$ points $x_{1}, \ldots, x_{k}$ and $y_{1}, \ldots, y_{l}$, where $N_{0}^{+} \cap N_{0}^{-}$is positive at $x_{j}$ and negative at $y_{j}$, in the sense of homology.

Then arguing as in $\$ 6$, we find that for small $t \in(0, \delta)$ we expect $k$ distinct, immersed SL homology 3-spheres $N_{t}$ diffeomorphic to $N_{0}^{+} \# N_{0}^{-}$, the connected sums of $N_{0}^{+}$and $N_{0}^{-}$at $x_{1}, \ldots, x_{k}$, and for small $t \in(-\delta, 0)$ we expect $l$ distinct, immersed SL homology 3 -spheres $N_{t}$ diffeomorphic to $N_{0}^{+} \# N_{0}^{-}$, the connected sums of $N_{0}^{+}$and $N_{0}^{-}$at $y_{1}, \ldots, y_{l}$.

Hence, as $\left[\Omega_{t}\right]$ passes through $W\left(\chi^{+}, \chi^{-}\right)$going from the negative to the positive side, we simultaneously create $k$ and destroy $l$ immersed special Lagrangian copies of $N_{0}^{+} \# N_{0}^{-}$. Suppose for simplicity that $N_{0}^{ \pm}$are the only SL homology 3-spheres in their homology classes $\chi^{ \pm}$. Then $I\left(\chi^{+}\right)=w\left(N_{0}^{+}\right)$ and $I\left(\chi^{-}\right)=w\left(N_{0}^{-}\right)$. Write $I\left(\chi^{+}+\chi^{-}\right)^{+}$for the value of $I\left(\chi^{+}+\chi^{-}\right)$at some point on the positive side of $W\left(\chi^{+}, \chi^{-}\right)$, and $I\left(\chi^{+}+\chi^{-}\right)^{-}$for its value at a nearby point on the negative side. Then we have

$$
I\left(\chi^{+}+\chi^{-}\right)^{+}-I\left(\chi^{+}+\chi^{-}\right)^{-}=(k-l) \cdot w\left(N_{0}^{+} \# N_{0}^{-}\right) .
$$

If the weight function $w$ satisfies the identity

$$
w\left(N_{0}^{+} \# N_{0}^{-}\right)=w\left(N_{0}^{+}\right) \cdot w\left(N_{0}^{-}\right) \text {for all homology 3-spheres } N_{0}^{ \pm},
$$


where multiplication is in the commutative ring $R$, then $(26)$ can be written

$$
I\left(\chi^{+}+\chi^{-}\right)^{+}-I\left(\chi^{+}+\chi^{-}\right)^{-}=\left(\chi^{+} \cap \chi^{-}\right) \cdot I\left(\chi^{+}\right) \cdot I\left(\chi^{-}\right),
$$

as $\chi^{+} \cap \chi^{-}=k-l$ and $w\left(N_{0}^{+} \# N_{0}^{-}\right)=w\left(N_{0}^{+}\right) \cdot w\left(N_{0}^{-}\right)=I\left(\chi^{+}\right) \cdot I\left(\chi^{-}\right)$. Because of the bilinearity of the r.h.s. of (28) in $I\left(\chi^{+}\right)$and $I\left(\chi^{-}\right)$, it is easy to see that (28) should also hold when there are finitely many SL homology 3 -spheres in $\chi^{ \pm}$, and not just one in each.

Thus, (28) gives a formula for how we expect $I\left(\chi^{+}+\chi^{-}\right)$to change as we pass through the hypersurface $W\left(\chi^{+}, \chi^{-}\right)$. The important thing about this formula is that the values of $I$ on the positive side of $W\left(\chi^{+}, \chi^{-}\right)$determine the values of $I$ on the negative side, and vice versa. So although $I$ is not invariant under deformations of $(M, J, \omega, \Omega)$ which alter the cohomology class $[\Omega]$, it transforms in a completely determined way, and that is more or less as useful.

In $\S 7.1$ we proposed the weight function $w(N)=\left|H_{1}(N, \mathbb{Z})\right|$, for $N$ a homology 3 -sphere. Now $H_{1}\left(N_{0}^{+} \# N_{0}^{-}, \mathbb{Z}\right) \cong H_{1}\left(N_{0}^{+}, \mathbb{Z}\right) \times H_{1}\left(N_{0}^{-}, \mathbb{Z}\right)$ as finite groups, and so

$$
\left|H_{1}\left(N_{0}^{+} \# N_{0}^{-}, \mathbb{Z}\right)\right|=\left|H_{1}\left(N_{0}^{+}, \mathbb{Z}\right)\right| \cdot\left|H_{1}\left(N_{0}^{-}, \mathbb{Z}\right)\right|
$$

when $N_{0}^{ \pm}$are homology 3-spheres. Thus this weight function $w$ satisfies (27), as we wish.

\subsection{Including multiple covers}

Let $N^{\prime}$ be an embedded SL homology 3-sphere in an almost Calabi-Yau 3-fold $(M, J, \omega, \Omega)$ with $\left[N^{\prime}\right]=\chi^{\prime} \in H_{3}(M, \mathbb{Z})$, and $\iota: N \rightarrow N^{\prime}$ be a finite cover of $N^{\prime}$ of degree $d>1$, and covering group $G$, so that $|G|=d$. Suppose $N$ is also a homology 3-sphere. Then we can regard $N$ as an immersed SL homology 3 -sphere in $M$, with $[N]=d\left[N^{\prime}\right]=d \chi^{\prime}$ in $H_{3}(M, \mathbb{Z})$. What contribution should $N$ make to $I\left(d \chi^{\prime}\right)$ ?

We claim that $N$ should contribute $w(N) / d$ to $I\left(d \chi^{\prime}\right)$, where $w$ is the weight function for embedded homology 3-spheres. Note that if $w$ takes values in $\mathbb{Z}$, then $w(N) / d$ takes values in $\mathbb{Q}$, so that our invariant $I\left(d \chi^{\prime}\right)$ will be actually be a rational number. Here is why. Let $N^{\prime \prime}$ be another SL homology 3 -sphere in $M$ intersecting $N^{\prime}$ transversely at one point, with $N^{\prime} \cap N^{\prime \prime}$ positive. Let $\left[N^{\prime \prime}\right]=\chi^{\prime \prime}$ in $H_{3}(M, \mathbb{Z})$. Deform $(M, J, \omega, \Omega)$ so that $[\Omega]$ passes through $W\left(\chi^{\prime}, \chi^{\prime \prime}\right)$ going from the negative to the positive side. 
Since $[N] \cap\left[N^{\prime \prime}\right]=d$ in homology, we expect from $\$ 7.2$ to create $d$ distinct new SL homology 3-spheres as we pass through $W\left(\chi^{\prime}, \chi^{\prime \prime}\right)$, all diffeomorphic to $N \# N^{\prime \prime}$.

However, a little thought shows that we actually create only one SL copy of $N \# N^{\prime \prime}$. Effectively this is because the internal symmetry group $G$ of $N$ with $|G|=d$ identifies the $d$ copies of $N \# N^{\prime \prime}$, so that they all give the same SL 3-fold. In order for (28) to give the correct transformation law for $I\left(d \chi^{\prime}+\chi^{\prime \prime}\right)$ across $W\left(\chi^{\prime}, \chi^{\prime \prime}\right)$, we must give $N$ the weight $w(N) / d$ rather than $w(N)$.

\subsection{Including SL 3-folds with stable singularities}

In $\$ 4.4$ we described a class of SL 3-folds with stable singularities. That is, in an almost Calabi-Yau 3-fold $(M, J, \omega, \Omega)$ one can have SL 3 -folds $N$ with one or more $T^{2}$-cone singularities, that persist under small deformations of $(J, \omega, \Omega)$. Should such singular SL 3 -folds also be counted in the invariant $I(\delta)$, with some appropriate topological weight?

I believe that the answer to this is yes. Furthermore, if the weight for nonsingular SL 3-folds $N$ is $w(N)=\left|H_{1}(N, \mathbb{Z})\right|$, then the weight for singular SL 3-folds may in some circumstances be negative. I have not yet sorted all the details of this out, and hope to do so in a future paper. However, here are the ideas which lead me to this conclusion.

In $\S 3-\delta$ and $\$ 5-\$ 6$ we described two kinds of 'index one' singularity of SL 3-folds, and analysed their topological effects. Now I know of at least two other kinds of 'index one' singularity, the first locally modelled on the SL 3-folds of [7, Ex. 7.4], and the second on the singularities described in [8, $\S 6]$. The topological effects are complicated to describe.

My calculations indicate that one thing that can happen with the first kind of index one singularity, is that one can simultaneously create, out of nothing, a nonsingular, immersed SL homology 3-sphere $N_{0}$ with one point of self-intersection $x$, and an embedded, singular SL 3 -fold $N_{1}$ with one $T^{2}$-cone singularity $y$, of the kind considered in $\$ 4.4$, and with $k^{a}=0$ for some $a=$ $1,2,3$.

In fact, we also create a family of nonsingular, embedded, diffeomorphic SL 3-folds $N_{t}$ for $t \in(0,1)$ with $b^{1}\left(N_{t}\right)=1$, which converge to $N_{0}$ as $t \rightarrow 0_{+}$ with local model $K_{\phi, A}$ near $x$, where $A \rightarrow 0_{+}$as $t \rightarrow 0_{+}$, and converge to $N_{1}$ as $t \rightarrow 1_{-}$with local model $L_{s}^{a}$ near $y$, where $s \rightarrow 0_{+}$as $t \rightarrow 1_{-}$. Then $N_{t}$ for $t \in[0,1]$ all have the same homology class $\left[N_{t}\right]=\chi \in H_{3}(M, \mathbb{Z})$. 
Now when it exists, $N_{0}$ contributes $w\left(N_{0}\right)$ to $I(\chi)$. In order for the invariant $I$ to be unchanged under this kind of transition we need $N_{1}$ to contribute $-w\left(N_{0}\right)$ to $I(\chi)$. It can be shown that $H_{1}\left(N_{0}, \mathbb{Z}\right) \cong H_{1}\left(N_{1}, \mathbb{Z}\right)$. Thus, if the weight for $N_{0}$ is $w\left(N_{0}\right)=\left|H_{1}\left(N_{0}, \mathbb{Z}\right)\right|$, as above, then the correct weight for $N_{1}$ is $w\left(N_{1}\right)=-\left|H_{1}\left(N_{1}, \mathbb{Z}\right)\right|$.

So I conjecture that for SL 3 -folds $N$ with one stable $T^{2}$-cone singularity of the kind considered in $\S 4.4$ and $k^{a}=0$ for some $a=1,2,3$, the appropriate weight is $w(N)=-\left|H_{1}(N, \mathbb{Z})\right|$. I am not yet sure of the answer when the $k^{a}$ are all nonzero, or there is more than one $T^{2}$-cone singularity.

\subsection{A preliminary conjecture}

I am now ready to formulate a first guess as to how to define an invariant counting special Lagrangian homology 3 -spheres, and what its properties should be under deformations of the underlying almost Calabi-Yau 3-fold.

Conjecture 7.3 Let $(M, J, \omega, \Omega)$ be a generic almost Calabi-Yau 3-fold. Then there exists $I: H_{3}(M, \mathbb{Z}) \rightarrow \mathbb{Q}$ with the following properties:

(a) For each $\delta \in H_{3}(M, \mathbb{Z})$, let $S(\delta)$ be the set of compact, immersed, possibly singular $S L 3$-folds $N$ in $M$, with $[N]=\delta$ and $b^{1}(N)=0$. Then $S(\delta)$ is finite, and

$$
I(\delta)=\sum_{N \in S(\delta)} w(N),
$$

where $w(N) \in \mathbb{Q}$ is a weight function depending only on the topology of $N$ and its immersion in $M$.

(b) Let $N$ be a nonsingular immersed SL 3-fold in $M$. Regard $N$ as a nonsingular, compact 3-manifold with immersion $\iota: N \rightarrow M$. Define $G(N)$ to be the group of diffeomorphisms $\phi: N \rightarrow N$ with $\iota \circ \phi=\iota$. Then $G(N)$ is finite, and the weight $w(N)$ is

$$
w(N)=\frac{\left|H_{1}(N, \mathbb{Z})\right|}{|G(N)|} .
$$

(c) I is unchanged by continuous deformations of $(M, J, \omega, \Omega)$ that change $[\omega]$ but leave $[\Omega]$ fixed, or multiply $[\Omega]$ by a nonzero complex number. Thus I depends only on the complex structure $J$ on $M$, and not on the metric $g$. 
(d) When we deform $(M, J, \omega, \Omega)$ so that $[\Omega]$ passes through one of the hypersurfaces $W\left(\chi^{+}, \chi^{-}\right)$in $H^{3}(M, \mathbb{C})$ given in Definition [7.9, the invariant I transforms according to a set of rules that we are not yet able to write down.

One of these rules should be that if $\chi^{+}$and $\chi^{-}$are primitive elements of $H_{3}(M, \mathbb{Z})$ and $I\left(\chi^{+}+\chi^{-}\right)^{ \pm}$are the values of $I\left(\chi^{+}+\chi^{-}\right)$at two nearby points on the positive and negative sides of $W\left(\chi^{+}, \chi^{-}\right)$, then

$$
I\left(\chi^{+}+\chi^{-}\right)^{+}-I\left(\chi^{+}+\chi^{-}\right)^{-}=\left(\chi^{+} \cap \chi^{-}\right) \cdot I\left(\chi^{+}\right) \cdot I\left(\chi^{-}\right) .
$$

Here are some remarks on this conjecture. Firstly, in defining GromovWitten invariants, $J$-holomorphic curves are counted with signs. But I believe that there is no corresponding way to define the sign of an SL 3-fold, and that SL 3-folds should be counted without signs.

Secondly, one effect of assuming that $(M, J, \omega, \Omega)$ is generic is that the only SL 3-folds that can exist in $M$ are those with stable singularities. So there is no need to restrict to SL 3 -folds with stable singularities in defining $S(\delta)$.

Thirdly, to understand how $I(\delta)$ transforms under deformations, it is enough to consider smooth 1-parameter families $\left\{\left(M, J_{t}, \omega_{t}, \Omega_{t}\right): t \in[0,1]\right\}$ of almost Calabi-Yau 3-folds, where $\left(M, J_{0}, \omega_{0}, \Omega_{0}\right)$ and $\left(M, J_{1}, \omega_{1}, \Omega_{1}\right)$ are generic (so that the invariant $I$ is defined when $t=0$ or 1 ) and the family itself is generic as a 1-parameter family.

Now by the ideas of $\delta 2.3$, the only singularities of SL 3-folds $N$ with $b^{1}(N)=0$ that we will encounter in such families of almost Calabi-Yau 3folds are singularities with index one. Thus, provided the general picture of $\S 2.3$ is correct, to prove Conjecture 7.3 we only need to know about special Lagrangian singularities with index one, and higher codimension singularities can be ignored.

Fourthly, in part (d) we have not given a complete set of rules for the transformation of $I$ on the hypersurfaces $W\left(\chi^{+}, \chi^{-}\right)$. Here is why. When we pass through the hypersurface $W\left(\chi^{+}, \chi^{-}\right)$we expect to create or destroy new SL 3 -folds with homology class $\chi^{+}+\chi^{-}$, which are connected sums of 3 -folds with homology classes $\chi^{+}$and $\chi^{-}$. But this is only the simplest kind of transition which happens on $W\left(\chi^{+}, \chi^{-}\right)$. 
For instance, if $N_{1}^{+}, N_{2}^{+} \in S\left(\chi^{+}\right)$and $N^{-} \in S\left(\chi^{-}\right)$, then on $W\left(\chi^{+}, \chi^{-}\right)$ we may create a new SL homology 3-sphere with homology class $2 \chi^{+}+\chi^{-}$, diffeomorphic to the triple connected sum $N_{1}^{+} \# N^{-} \# N_{2}^{+}$. So there should be some change to $I\left(2 \chi^{+}+\chi^{-}\right)$on $W\left(\chi^{+}, \chi^{-}\right)$. Similarly, if $a, b$ are positive integers then we can try and take a multiple connected sum of $a$ elements of $S\left(\chi^{+}\right)$and $b$ elements of $S\left(\chi^{-}\right)$to get a new SL homology 3-sphere with homology class $a \chi^{+}+b \chi^{-}$.

However, there is a problem: can we include an SL 3-fold in such a connected sum more than once, and if so, how? The answer to this appears to be rather complex, and I do not yet understand it, which is why I'm not ready to write down a full set of transformation rules for $I$.

\subsection{Relationships with String Theory}

I now want to argue that the invariant $I$ postulated above should have an interpretation in String Theory, and may fit into a piece of the Mirror Symmetry story for Calabi-Yau 3-folds which is not understood at present. There are two main reasons for this:

(a) The invariant $I$ counts objects which are significant in String Theory, namely isolated 3-branes; and

(b) Mirror Symmetry strongly suggests that for a Calabi-Yau 3-fold $X$ there should be discrete, $\mathbb{Q}$-valued invariants defined on $H_{3}(X, \mathbb{Z})$ or something like it, which are related under the mirror transform to the Gromov-Witten invariants of the mirror manifold $Y$. But no such invariants are known at present.

We first discuss reason (a). In String Theory, SL 3-folds correspond roughly to physical objects called 3-branes. But a 3 -brane is not just a 3-dimensional submanifold $N$; it also carries with it a complex line bundle over $N$ with a flat U(1)-connection. (For a discussion of this, see for instance Strominger, Yau and Zaslow [16].) We will call a 3-brane isolated if it admits no deformations, which happens when $N$ is a rational homology 3-sphere.

If $N$ is a compact 3-manifold, then flat $\mathrm{U}(1)$-connections on $N$ are equivalent to group homomorphisms $H_{1}(N, \mathbb{Z}) \rightarrow \mathrm{U}(1)$. But when $H_{1}(N, \mathbb{Z})$ is finite, it is easy to show using the theory of finite abelian groups that the number of group homomorphisms $H_{1}(N, \mathbb{Z}) \rightarrow \mathrm{U}(1)$ is exactly $\left|H_{1}(N, \mathbb{Z})\right|$. Hence, if $N$ is a special Lagrangian homology 3 -sphere, then there are exactly 
$\left|H_{1}(N, \mathbb{Z})\right|$ flat $\mathrm{U}(1)$-connections over $N$, and so $N$ gives rise to $\left|H_{1}(N, \mathbb{Z})\right|$ isolated 3-branes.

Therefore, for the case of nonsingular, embedded SL 3-folds, the invariant $I(\delta)$ discussed above counts the number of isolated 3-branes in the homology class $\delta$. So it is a natural thing to count from the String Theory point of view, although the author formulated Conjecture 7.3 without knowing this.

Next we discuss reason (b). The following basic outline of Mirror Symmetry in String Theory is by now well known, and is described for instance in Greene and Plesser [3]. To a pair $(X, S)$, where $X$ is a Calabi-Yau 3-fold and $S$ some 'extra structure' which we will not worry about, a physicist associates a Super Conformal Field Theory (SCFT). There are believed to exist 'mirror pairs' $(X, S)$ and $(Y, T)$ whose SCFT's are isomorphic under a simple involution of the SCFT structure.

If $(X, S)$ and $(Y, T)$ are such a mirror pair, then $H^{2,1}(X) \cong H^{1,1}(Y)$, and certain cubic forms $I_{X}^{2,1}$ on $H^{2,1}(X)$ and $I_{Y}^{1,1}$ on $H^{1,1}(Y)$ agree. Here $I_{X}^{2,1}$ has a simple definition, depending on the variation of complex structures on $X$. However, $I_{Y}^{1,1}$ has a complicated definition, involving an infinite sum over homology classes $\alpha$ in $H_{2}(Y, \mathbb{Z})$ of the 'number of rational curves' in $Y$ with homology class $\alpha$, multiplied by complex functions of a standard form.

Now, here is the important point. If we accept the mirror conjecture, then $I_{X}^{2,1}$ can be written as essentially the sum of a power series with integer (or rational) coefficients. Is there a way of understanding, purely in terms of $X$ and without introducing the mirror $Y$, why these numbers should be integers, and what they mean?

The author hopes that this question can be answered as follows. There should be a way to write $I_{X}^{2,1}$ as a sum over finite collections of elements of $H_{3}(X, \mathbb{Z})$ of integer or rational invariants similar to the invariant $I$ described in $\$ 7.5$, multiplied by complex (holomorphic, in a suitable sense) functions of $[\Omega]$ of a standard form. As $[\Omega]$ passes through a hypersurface $W\left(\chi^{+}, \chi^{-}\right)$, these complex functions may change discontinuously to compensate for changes in $I$, so that $I_{X}^{2,1}$ remains continuous. The reason the numbers in the series are integers (or rationals) is then that they count collections of SL 3-folds satisfying certain conditions.

\section{References}


[1] A. Butscher, Regularizing a Singular Special Lagrangian Variety, math.DG/0110053, 2001.

[2] K. Fukaya, Y.-G. Oh, H. Ohta and K. Ono, Lagrangian intersection Floer theory - anomaly and obstruction, preprint, 2000.

[3] B.R. Greene and M.R. Plesser, An Introduction to Mirror Manifolds, pages 1-27 in S.-T. Yau, editor, Essays on Mirror Manifolds, International Press, Hong Kong, 1992.

[4] F.R. Harvey, Spinors and calibrations. Academic Press, San Diego, 1990.

[5] F.R. Harvey and H.B. Lawson, Calibrated geometries, Acta Math. 148 (1982), 47-157.

[6] D.D. Joyce, Special Lagrangian $m$-folds in $\mathbb{C}^{m}$ with symmetries, math.DG/0008021, 2000. To appear in the Duke Mathematical Journal.

[7] D.D. Joyce, Constructing special Lagrangian $m$-folds in $\mathbb{C}^{m}$ by evolving quadrics, Math. Ann. 320 (2001), 757-797. math.DG/0008155.

[8] D.D. Joyce, Evolution equations for special Lagrangian 3-folds in $\mathbb{C}^{3}$, math.DG/0010036, 2000. To appear in the Annals of Global Analysis and Geometry.

[9] D.D. Joyce, Singularities of special Lagrangian fibrations and the SYZ Conjecture, math.DG/0011179, 2000.

[10] D.D. Joyce, Ruled special Lagrangian 3-folds in $\mathbb{C}^{3}$, math.DG/0012060, 2000. To appear in the Proceedings of the London Mathematical Society.

[11] D.D. Joyce, Special Lagrangian 3-folds and integrable systems, math.DG/0101249, 2001. To appear in volume 1 of the Proceedings of the Mathematical Society of Japan's 9th International Research Institute on Integrable Systems in Differential Geometry, Tokyo, 2000.

[12] D.D. Joyce, Lectures on Calabi-Yau and special Lagrangian geometry, math.DG/0108088, 2001.

[13] G. Lawlor, The angle criterion, Inv. math. 95 (1989), 437-446. 
[14] D. McDuff and D. Salamon, J-holomorphic Curves and Quantum Cohomology. A.M.S., Rhode Island, 1994.

[15] R.C. McLean, Deformations of calibrated submanifolds, Communications in Analysis and Geometry 6 (1998), 705-747.

[16] A. Strominger. S.-T. Yau and E. Zaslow, Mirror Symmetry is T-duality, Nucl. Phys. B479 (1996), 243-259. hep-th/9606040. 